\title{
On horizons and plane waves
}

\author{
James T Liu ${ }^{1,3}$, Leopoldo A Pando Zayas ${ }^{1,2}$ and Diana Vaman ${ }^{3}$ \\ ${ }^{1}$ Michigan Center for Theoretical Physics, University of Michigan, Ann Arbor, \\ MI 48109-1120, USA \\ ${ }^{2}$ School of Natural Sciences, Institute for Advanced Study, Princeton, NJ 08540, USA \\ ${ }^{3}$ Department of Physics, Princeton University, Princeton, NJ 08544, USA
}

Received 9 April 2003, in final form 31 July 2003

Published 12 September 2003

Online at stacks.iop.org/CQG/20/4343

\begin{abstract}
We investigate the possibility of having an event horizon within several classes of metrics that asymptote to the maximally supersymmetric IIB plane wave. We show that the presence of a null Killing vector (not necessarily covariantly constant) implies an effective separation of the Einstein equations into a standard and a wave component. This feature may be used to generate new supergravity solutions asymptotic to the maximally supersymmetric IIB plane wave, starting from standard seed solutions such as branes or intersecting branes in flat space. We find that in many cases it is possible to preserve the extremal horizon of the seed solution. On the other hand, non-extremal deformations of the plane wave solution result in naked singularities. More generally, we prove a no-go theorem against the existence of horizons for backgrounds with a null Killing vector and which contain at most null matter fields. Further attempts at turning on a non-zero Hawking temperature by introducing additional matter have proved unsuccessful. This suggests that one must remove the null Killing vector in order to obtain a horizon. We provide a perturbative argument indicating that this is in fact possible.
\end{abstract}

PACS numbers: 11.25.-w, 04.70.Bw

\section{Introduction}

Black holes play a special role in our understanding of nature: arising as classical solutions to gravity equations they pose challenging questions at the quantum level. In the framework of general relativity the best understood situations pertain to asymptotically flat black holes. Motivated by the AdS/CFT correspondence [1] interest has been directed to the study of black holes that asymptote to AdS. These black holes have properties that fundamentally distinguish them from asymptotically flat black holes, like the possibility of a positive specific heat. Some of the properties of the simplest black hole in this class - the Schwarzschild-AdS black hole-were studied in [2]. Most of the properties of asymptotically AdS black holes 
have received interesting interpretations in the framework of the AdS/CFT correspondence. In particular, Witten argued that the Hawking-Page phase transition is the supergravity dual effect to the confinement/deconfinement phase transition for gauge theories on the sphere [3]. Other properties of black holes asymptoting to AdS have been discussed in the literature (see, for example, [4]).

Recently Berenstein, Maldacena and Nastase (BMN) [5] have proposed a correspondence between a limit of the $\mathrm{AdS}_{5} \times S^{5}$ IIB background and a subsector of operators of $\mathcal{N}=4$ super Yang-Mills. The limit on the IIB supergravity side is the maximally supersymmetric plane wave background with constant null RR 5-form (the BFHP solution) [6]

$$
\begin{aligned}
& \mathrm{d} s^{2}=-4 \mathrm{~d} x^{+} \mathrm{d} x^{-}-\frac{1}{16} \mu^{2} z_{i} z^{i}\left(\mathrm{~d} x^{+}\right)^{2}+\mathrm{d} z_{i} \mathrm{~d} z^{i}, \\
& F_{+1234}=F_{+5678}=\mu .
\end{aligned}
$$

This solution is the analogue of the maximally supersymmetric plane wave of 11-dimensional (11D) supergravity [7].

One of the natural questions that arises from the BMN construction relates to the nonzero temperature deformation of the correspondence, much in the same way as the AdS/CFT correspondence extends to non-zero temperature [3]. On the string theory side some progress in formulating the correspondence has been achieved. Exploiting the fact that the maximally supersymmetric IIB plane wave is exactly solvable in the light-cone gauge [8,9], the thermal partition function has been calculated and shown to display a Hagedorn temperature depending on the parameter $\mu$ above [10]. This nontrivial dependence of the Hagedorn temperature makes the possibility of a phase transition even more tantalizing. Many natural questions, however, remained unanswered. In particular, the structure of the analogue of the Schwarzschild-AdS black hole in the context of plane waves is not known. More generally: what are the properties of black holes that asymptote to the homogeneous plane wave spacetime?

The question of the Schwarzschild black hole asymptoting to the BFHP plane wave has been addressed from different angles [11-14]. In [11, 12] it was made explicit that the answer cannot come from a Penrose limit of the Schwarzschild-AdS black hole. This was further elaborated upon in [13] where it was also shown that the existence of a covariantly constant null Killing vector is an obstruction to having an event horizon. In [14] a further study of asymptotically plane wave spacetimes was carried out and some solutions were presented using the Garfinkle-Vachaspati method [15] (see also [16]).

In this paper, we investigate various possibilities for introducing event horizons in geometries that asymptote to the BFHP plane wave. We consider two fundamentally different classes of solutions: extremal and non-extremal. More precisely, we describe solutions with zero Hawking temperature and attempt to generalize them to solutions with non-zero Hawking temperature.

To construct the first class of solutions, that is, zero temperature black objects containing an event horizon and asymptoting to the BFHP plane wave, we make use of a separation property of backgrounds with a null Killing vector. Namely, we consider metrics that generalize the BFHP plane wave while maintaining a null Killing vector,

$$
\mathrm{d} s^{2}=\mathrm{e}^{2 A}\left[-4 \mathrm{~d} x^{+} \mathrm{d} x^{-}+\mathcal{H} \mathrm{d} x^{+2}\right]+\sum_{i} \mathrm{e}^{2 B_{i}} \mathrm{~d} z_{i}^{2},
$$

where $A(\vec{z}), B_{i}(\vec{z})$ and $\mathcal{H}(\vec{z})$ are in general functions of all transverse coordinates $\vec{z}$. A crucial observation that may be used to establish various properties is the fact that the Ricci tensor decomposes [15] into standard and plane wave components, 


$$
R_{M N}=R_{M N}^{(0)}-\frac{1}{2} \delta_{M}^{+} \delta_{N}^{+} \mathrm{e}^{2 A} \sum_{i} \mathrm{e}^{-2 B_{i}}\left[\partial_{i} \partial_{i} \mathcal{H}+\partial_{i} \mathcal{H} \partial_{i} \mathcal{G}_{i}\right]
$$

where $R_{M N}^{(0)}$ is computed from (1.2) when $\mathcal{H}=0$ and $\mathcal{G}_{i}=2 A+\sum_{j} B_{j}-2 B_{i}$.

A very natural way to exploit the property (1.3) is to use it as a solution generating mechanism. Given that only the $R_{++}$component of the Ricci tensor is modified, this allows for the possibility of 'superposing' a plane wave on top of a given solution. Namely, finding a suitable matter contribution $\delta T_{++}$we can generalize a given supergravity solution with $\mathcal{H}=0$ to a new solution with $\mathcal{H} \neq 0$. Imposing that asymptotically $A, B \longrightarrow 0$ and that $\mathcal{H}$ approaches the form of (1.1) we construct solutions that asymptote to the BFHP plane wave.

Along the lines of the solution generating point of view we can reinterpret the BFHP solution as the $\mathcal{H}$-deformation of flat space. Under this scope we review the construction of D-branes and more importantly, we obtain new solutions corresponding to $\mathcal{H}$-deforming intersecting branes. We find it possible in some cases to arrange for the $\mathcal{H}$-deformation to keep the regular horizon characteristic of the seed solutions while changing the solutions asymptotically to that of BFHP for large $\vec{z}$. This way we present a class of solutions that asymptote to the BFHP plane wave and have regular event horizons.

We then attempt to further generalize such solutions to include an event horizon corresponding to non-zero Hawking temperature. We study possible generalizations under various symmetry requirements. In particular we consider $S O(8)$ and $S O(4) \times S O(4)$ generalizations of the BFHP solution. We find that in the presence of a null Killing vector such generalizations yield singular solutions. Using the separation property of the Ricci tensor (1.3), we show that generalizations of the BFHP solution keeping a null Killing vector and with arbitrary splitting of the transverse directions cannot lead to a smooth horizon. Following the expectations raised in the zero temperature case we go on to consider non-zero Hawking temperature generalizations of the $\mathcal{H}$-deformed branes and intersecting brane solutions. We find however, that all such generalizations yield singular event horizons.

Having identified the main obstruction to having a non-zero Hawking temperature event horizon as the presence of a null Killing vector, we consider a perturbative argument in favour of the existence of such solutions once the null Killing vector is removed. Namely, we consider the black string in ten dimensions and discuss the conditions for turning on an RR 5-form. We find, neglecting backreaction, compatibility between the Bianchi identity and the asymptotic conditions. Moreover, we show explicitly that the backreaction is under control.

Because of our interest in identifying regular event horizons, we begin in section 2 with an analysis of the horizon structure in generalized plane wave backgrounds. In section 3 , we exploit the separation property of the Ricci tensor to construct new multiple charge solutions with zero temperature, including a deformation of the well-known D1-D5 system. Then, in section 4 , we investigate the possibility of finding solutions with non-zero temperature. We find that the presence of a null Killing vector presents an obstruction to the existence of such solutions. This leads us to a no-go theorem proved in section 5 . In section 6 , we consider turning on additional sources (while maintaining a null Killing vector), but show that such examples are unable to overcome the no-go theorem. Finally, we conclude in section 7 by demonstrating (at least perturbatively) how a horizon may be obtained by relaxing the null Killing vector condition.

\section{Asymptotic time and event horizons in plane waves}

Since our main endeavour is dedicated to finding supergravity solutions that share the same asymptotics as the maximally supersymmetric IIB plane wave and that have a regular event 
horizon, we devote this section to examining the criteria for the existence of an event horizon. We also discuss how different asymptotics might change the analysis of the event horizon. First, a cautionary word about event horizons in asymptotically non-flat spaces is in order. A rigorous definition of event horizons in the case of asymptotically flat black holes is given, for example, in [17] (pp 299-300). Namely, a black hole $B$ is defined as a region of a strongly asymptotically predictable spacetime $\left(M, g_{a b}\right)$ given by $B=\left[M-J^{-}\left(\mathcal{J}^{+}\right)\right]$. This definition is equivalent under very mild topological conditions to $B \cap J^{-}\left(\mathcal{J}^{+}\right)=\emptyset$, where $J^{-}\left(\mathcal{J}^{+}\right)$ denotes the causal past of the future null infinity. However, such a definition is lacking for general asymptotics, in part because of the difficulties in identifying $\mathcal{J}^{+}$. (Discussions of the causal structure in spaces asymptoting to plane wave have appeared in [18-20].) The need for finding criteria for an event horizon without requiring knowledge of the global development of a spacetime was addressed partially in [17] (pp 308-12). Along those lines we will, as a working definition, declare a black hole to be a region causally disconnected from asymptotic infinity.

Our analysis, however, uses asymptotic infinity rather indirectly by replacing it by a suitable causally connected region $R$. Thus, we only need to show that this region $R$, which is causally connected to asymptotic infinity, is causally disconnected from the black hole region. Therefore, our analysis boils down to the study of the proper (affine) time and the time of propagation of signals along timelike and null geodesics. Namely, we require the proper (affine) time to be finite so that the spacetime can be extended beyond that point but we require the (coordinate) time travelled along a timelike or null geodesic to be infinite so that causal communication is impossible. Note that, by coordinate time, we mean a suitable time coordinate conjugate to a timelike Killing vector. Up to finite time dilation, this would be the time measured by a static observer in the region $R$.

Of course, it may be the case that there is not a unique timelike Killing vector. In this case, different choices may be made for coordinate time, corresponding to different observers boosted with regard to each other. For finite boosts, all such observers are in causal contact. Thus if any single observer (i.e., choice of time coordinate) can communicate with the purported black hole region, then a horizon cannot exist and the 'black hole' would instead be a singularity of some sort (assuming a curvature singularity develops). As a result, our procedure is clear. To demonstrate the existence of a horizon, we must show that all possible choices of coordinate time yield an infinite $\Delta t$ along the geodesic. In practice, however, it is often sufficient to consider only a handful of choices, as will be made clear below.

For the geometries we are interested in, it is sufficient to discuss only metrics which admit a null Killing vector. Let us begin by considering a generalization of the plane wave metric of the form

$$
\mathrm{d} s^{2}=\mathrm{e}^{2 A(r)}\left[-4 \mathrm{~d} x^{+} \mathrm{d} x^{-}+\mathcal{H} \mathrm{d} x^{+2}\right]+\mathrm{e}^{2 B(r)} \mathrm{d} r^{2}+\cdots,
$$

where ellipses signify other coordinates which are unimportant for the present purpose. In addition, let us assume that there is an event horizon at some finite distance $r_{0}$. Then one can study geodesics crossing the horizon and estimate the affine time and the time as measured by an observer in causal contact with infinity. To investigate the conditions under which an event horizon might exist, we consider timelike and null radial geodesics in this geometry. The geodesic equations can be obtained from the following effective Lagrangian,

$$
\mathcal{L}=\mathrm{e}^{2 A(r)}\left[-4 \dot{x}^{+} \dot{x}^{-}+\mathcal{H} \dot{x}^{+2}\right]+\mathrm{e}^{2 B(r)} \dot{r}^{2},
$$

where dots indicate differentiation with respect to the proper time. Since the above Lagrangian does not depend explicitly on $x^{+}$and $x^{-}$, we have two integrals of motion, $E_{+}$and $E_{-}$, which allow us to solve for the motion along $x^{+}$and $x^{-}$as

$$
\dot{x}^{+}=\mathrm{e}^{-2 A} E_{-}, \quad \dot{x}^{-}=\frac{1}{2} \mathrm{e}^{-2 A}\left(\mathcal{H} E_{-}-E_{+}\right) .
$$


We set $\mathcal{L}=-1,0$ for timelike and null geodesics respectively and solve for motion along the radial coordinate

$$
\dot{r}^{2}=\mathrm{e}^{-2 B}\left[\mathrm{e}^{-2 A} E_{-}\left(\mathcal{H} E_{-}-2 E_{+}\right)+\mathcal{L}\right] .
$$

By integrating this equation we find the proper time needed to travel between two radial positions $r_{\text {in }}$ and $r_{\text {fin }}$ along an incoming geodesic

$$
\Delta \tau=\int_{r_{\text {fin }}}^{r_{\text {in }}} \mathrm{d} r \mathrm{e}^{B}\left[\mathrm{e}^{-2 A} E_{-}\left(\mathcal{H} E_{-}-2 E_{+}\right)+\mathcal{L}\right]^{-1 / 2} .
$$

Similarly, we have the following formal variations of $x^{+}$and $x^{-}$:

$$
\begin{aligned}
& \Delta x^{+}=E_{-} \int_{r_{\text {fin }}}^{r_{\text {in }}} \mathrm{e}^{-2 A+B}\left[\mathrm{e}^{-2 A} E_{-}\left(\mathcal{H} E_{-}-2 E_{+}\right)+\mathcal{L}\right]^{-1 / 2} \mathrm{~d} r, \\
& \Delta x^{-}=\frac{1}{2} \int_{r_{\text {fin }}}^{r_{\text {in }}} \mathrm{e}^{-2 A+B}\left(\mathcal{H} E_{-}-E_{+}\right)\left[\mathrm{e}^{-2 A} E_{-}\left(\mathcal{H} E_{-}-2 E_{+}\right)+\mathcal{L}\right]^{-1 / 2} \mathrm{~d} r .
\end{aligned}
$$

To identify an asymptotic time coordinate for the metric (2.1), we note that it admits two Killing vectors, $\partial / \partial x^{+}$and $\partial / \partial x^{-}$. Thus we may choose to measure time as

$$
\Delta t=c^{+} \Delta x^{+}+c^{-} \Delta x^{-}
$$

(with constant $c^{+}$and $c^{-}$), so long as $\Delta t$ is a timelike interval. This results in the condition $c^{+}\left(c^{+} \mathcal{H}-4 c^{-}\right)<0$. So long as we are interested in solutions that asymptote to the maximally supersymmetric IIB plane wave, then

$$
\mathcal{H} \stackrel{r \rightarrow \infty}{\longrightarrow}-\frac{1}{16} \mu^{2} r^{2}
$$

so that $\partial / \partial x^{+}$by itself is a timelike Killing vector. In this case $x^{+}$can be taken as a natural asymptotic time coordinate, and we may set $c^{-}=0$ in (2.7). However, for $\partial / \partial x^{+}$either null or spacelike, we cannot set $c^{-}=0$, and hence must resort to $x^{-}$(or some combination of $x^{+}$and $x^{-}$) as an asymptotic time coordinate. We will make a distinction between these two possibilities below.

Since we are interested in motion near the purported horizon at $r_{0}$, a generic behaviour for the warp factors can be modelled by

$$
\mathrm{e}^{2 A} \sim\left(r-r_{0}\right)^{2 a}, \quad \mathrm{e}^{2 B} \sim\left(r-r_{0}\right)^{2 b} .
$$

The behaviour of the function $\mathcal{H}$ can be a little more involved. Let us assume a power-like behaviour:

$$
\mathcal{H} \sim\left(r-r_{0}\right)^{2 h} \operatorname{sgn} \mathcal{H} .
$$

For (2.5) and (2.6) to make sense we need the square root to be real. We first consider the case $\mathcal{H} \rightarrow$ const at the horizon, i.e., $h \geqslant 0$. Taking $E_{+}<0$ we find

$$
\Delta \tau \sim \int_{0}^{\epsilon} \mathrm{d} x x^{a+b}, \quad \Delta x^{+} \sim \int_{0}^{\epsilon} \mathrm{d} x x^{b-a}, \quad \Delta x^{-} \sim \int_{0}^{\epsilon} \mathrm{d} x x^{b-a} .
$$

In this case, regardless of the choice of time coordinate, (2.7), this means that to have a horizon we need $a+b>-1$ and $b-a \leqslant-1$. On the other hand, if $\mathcal{H}$ grows $(h<0)$, we need $\operatorname{sgn} \mathcal{H}=1$ (otherwise we would encounter a repulsive singularity much like in the negative mass Schwarzschild black hole). In this case,

$$
\Delta \tau \sim \int_{0}^{\epsilon} \mathrm{d} x x^{a+b+|h|}, \quad \Delta x^{+} \sim \int_{0}^{\epsilon} \mathrm{d} x x^{b-a+|h|}, \quad \Delta x^{-} \sim \int_{0}^{\epsilon} \mathrm{d} x x^{b-a-|h|} .
$$

Here care must be taken in relating asymptotic time to $x^{+}$and $x^{-}$. Note that $\Delta x^{-}$is always strictly more divergent than $\Delta x^{+}$since $|h|>0$. Hence the shortest attainable $\Delta t$ is given 
by using $x^{+}$as asymptotic time, which is only possible for $\partial / \partial x^{+}$timelike. Since all other observers would measure a longer time interval, we only need to check that $\Delta x^{+}$diverges. As a result, the horizon conditions are $a+b+|h|>-1$ and $b-a+|h| \leqslant-1$. On the other hand, if $\partial / \partial x^{+}$were null or spacelike, $\Delta t$ would always include a non-vanishing contribution from $\Delta x^{-}$. In this case, the second horizon condition is modified to $b-a-|h| \leqslant-1$, which is a weaker condition than that for $\partial / \partial x^{+}$timelike. Finally, let us note that for a logarithmic behaviour, $\mathcal{H} \sim\left|\log \left(r-r_{0}\right)\right| \operatorname{sgn} \mathcal{H}$, the situation is effectively identical to the $h \geqslant 0$ case. The only novelty is that $\operatorname{sgn} \mathcal{H}=-1$ leads to a repulsive, negative mass type of behaviour.

To summarize, for solutions that asymptote to the maximally supersymmetric IIB plane wave, in which case $\partial / \partial x^{+}$is timelike, the conditions for the existence of a horizon are

$$
\begin{array}{lll}
a+b>-1, & a-b \geqslant 1 & \text { if } \quad h \geqslant 0 ; \\
a+b+|h|>-1, & a-b \geqslant 1+|h| & \text { if } \quad h<0 \text { and } \operatorname{sgn} \mathcal{H}=+1 ; \\
a+b>-1, & a-b \geqslant 1 & \text { if } \quad \mathcal{H} \sim\left|\log \left(r-r_{0}\right)\right|,
\end{array}
$$

where $a, b$ and $h$ have been defined in (2.9) and (2.10), respectively. Note that the second line is stronger than the first line. Hence if no horizon is possible for $h \geqslant 0$, neither can it be possible for $h<0$.

More generally, one can state that the criteria (2.13) apply for any background whose asymptotics are those of a homogeneous plane wave, i.e., $H \stackrel{r \rightarrow \infty}{\longrightarrow}-\mu_{i j}^{2} x^{i} x^{j}$. Since $R_{++}$is positive by the weak energy condition, and $R_{++}$is the sum of the eigenvalues of $\mu_{i j}^{2}$, this implies that $\partial / \partial x^{+}$is asymptotically a timelike Killing vector; therefore all results previously derived are valid. On the other hand, for the case where $\partial / \partial x^{+}$is null or spacelike, our above analysis still holds, provided the second condition in (2.13) is replaced by

$a+b+|h|>-1, \quad a-b \geqslant 1-|h| \quad$ if $\quad h<0 \quad$ and $\quad \operatorname{sgn} \mathcal{H}=+1$.

This condition is now weaker than for the $h \geqslant 0$ case.

The set of conditions for the existence of a horizon defined as the boundary of a region causally disconnected from infinity, (2.13), can be equally well derived from a local analysis of the horizon. In the latter case we define the horizon as the boundary of a region of no escape; the idea behind this local definition is that gravity bends light, and a massive enough object could ultimately trap the light wavefronts. If this were to happen, then the outgoing light wavefronts would have a decreasing area, finally reaching the horizon whereupon the light rays would be instantaneously parallel. Since the rate of change in the area is given by the scalar expansion $\theta$, we turn to a computation of the expansions associated with the two null vectors pointing in the directions of the incoming and outgoing light wavefronts. For instance, the expansion associated with the the vector tangent to the null geodesic $k^{\mu}=\left(\dot{x}^{+}, \dot{x}^{-}, \dot{r}, \overrightarrow{0}\right)$ is $\theta_{k}=\widehat{D_{\mu} k^{\mu}}$ where the hat denotes the implicit projection onto the $d-2$ vector space orthogonal to the null vectors. The other null vector is normalized by $l^{\mu} k_{\mu}=-1$, and since it corresponds to the outgoing light wavefront it has a non-vanishing radial component. The trapping horizon is the closure of a hypersurface foliated by marginal surfaces, on which $\theta_{l}$ vanishes and $\theta_{k}$ is non-vanishing and negative (see, for example, [21]). Therefore in the trapped region we have $\theta_{k} \theta_{l}>0$. Without going into further detail, one obtains the weaker of the two conditions in (2.13) from the requirement that the second expansion is non-zero and negative at the horizon, while the strongest condition (the fact that infinity and the interior of the horizon are causally disconnected) is equivalent to the fact that the first expansion (the $\theta_{l}$ one) vanishes. 


\section{Solution generation with a null Killing vector}

Having examined the criteria for the existence of a regular event horizon in a generalized plane wave metric, we now turn to some examples. Since a covariantly constant null Killing vector precludes the existence of a regular horizon, here we relax the covariant constancy but maintain a null Killing vector. In this case, we may apply the observation of Garfinkle and Vachaspati [15] (see also [16]) that such a metric with a null Killing direction gives rise to a separation property of the Ricci tensor, (1.3). As indicated below, this property may be exploited to construct new wave solutions, given an initial 'seed' solution.

\subsection{The Ricci tensor in the presence of a null Killing vector}

While the separation property of $[15,16]$ is more general than that considered here, for the applications considered in this paper, it is sufficient to take a generic metric of the form

$$
\mathrm{d} s^{2}=\mathrm{e}^{2 A}\left[-4 \mathrm{~d} x^{+} \mathrm{d} x^{-}+\mathcal{H} \mathrm{d} x^{+2}\right]+\sum_{i} \mathrm{e}^{2 B_{i}} \mathrm{~d} z_{i}^{2},
$$

where $A(\vec{z}), B_{i}(\vec{z})$ and $\mathcal{H}(\vec{z})$ are in general functions of all transverse coordinates $\vec{z}$. Note that this form of the metric allows us to subsequently split the $z_{i}$ directions into additional longitudinal, relative-transverse and overall-transverse directions by taking the functions to be independent of some of the $z_{i}$.

For this metric, the non-vanishing Christoffel symbols are

$$
\begin{aligned}
& \Gamma^{+}{ }_{+i}=\partial_{i} A, \quad \Gamma^{-}{ }_{-i}=\partial_{i} A, \quad \Gamma_{+i}^{-}=-\frac{1}{4} \partial_{i} \mathcal{H}, \\
& \Gamma^{i}{ }_{++}=-g_{++} \mathrm{e}^{-2 B_{i}}\left(\partial_{i} A+\frac{1}{2} \partial_{i} \log \mathcal{H}\right), \quad \Gamma^{i}{ }_{+-}=-g_{+-} \mathrm{e}^{-2 B_{i}} \partial_{i} A, \\
& \Gamma^{i}{ }_{j k}=\delta_{i j} \partial_{k} B_{i}+\delta_{i k} \partial_{j} B_{i}-\mathrm{e}^{2 B_{j}-2 B_{i}} \delta_{j k} \partial_{i} B_{j} .
\end{aligned}
$$

In this expression and below, indices are not summed over unless explicitly indicated. A straightforward computation yields for the nontrivial components of the Ricci tensor

$$
\begin{gathered}
R_{+-}=-g_{+-} \sum_{i} \mathrm{e}^{-2 B_{i}}\left[\partial_{i} \partial_{i} A+\partial_{i} A \partial_{i} \mathcal{G}_{i}\right] \\
R_{++}=-g_{++} \sum_{i} \mathrm{e}^{-2 B_{i}}\left[\partial_{i} \partial_{i} A+\partial_{i} A \partial_{i} \mathcal{G}_{i}\right]-\frac{1}{2} \mathrm{e}^{2 A} \sum_{i} \mathrm{e}^{-2 B_{i}}\left[\partial_{i} \partial_{i} \mathcal{H}+\partial_{i} \mathcal{H} \partial_{i} \mathcal{G}_{i}\right], \\
R_{i j}=-\frac{1}{2} \partial_{i} \partial_{j}\left(\mathcal{G}_{i}+\mathcal{G}_{j}\right)-2 \partial_{i} A \partial_{j} A+2\left(\partial_{i} A \partial_{j} B_{i}+\partial_{j} A \partial_{i} B_{j}\right) \\
+\sum_{k}\left(\partial_{i} B_{j} \partial_{j} B_{k}+\partial_{j} B_{i} \partial_{i} B_{k}\right)-\sum_{k} \partial_{i} B_{k} \partial_{j} B_{k}-2 \partial_{i} B_{j} \partial_{j} B_{i} \\
\quad-g_{i j} \sum_{k} \mathrm{e}^{-2 B_{k}}\left[\partial_{k} \partial_{k} B_{i}+\partial_{k} B_{i} \partial_{k} \mathcal{G}_{k}\right]
\end{gathered}
$$

where $\mathcal{G}_{i}=2 A+\sum_{j} B_{j}-2 B_{i}$. Note, in particular, that $\mathcal{H}$ only appears in $R_{++}$. As a result, this allows us to write

$$
R_{M N}=R_{M N}^{(0)}-\frac{1}{2} \delta_{M}^{+} \delta_{N}^{+} \mathrm{e}^{2 A} \sum_{i} \mathrm{e}^{-2 B_{i}}\left[\partial_{i} \partial_{i} \mathcal{H}+\partial_{i} \mathcal{H} \partial_{i} \mathcal{G}_{i}\right],
$$

where $R_{M N}^{(0)}$ is computed from (3.1) when $\mathcal{H}=0$. Note that, up to a suitable coordinate transformation, $R_{M N}^{(0)}$ is simply the Ricci tensor for the 'conventional' metric

$$
\mathrm{d} s^{2}=\mathrm{e}^{2 A}\left[-\mathrm{d} t^{2}+\mathrm{d} w^{2}\right]+\sum_{i} \mathrm{e}^{2 B_{i}} \mathrm{~d} z_{i}^{2} .
$$


A particular case of (3.1) that we will often be interested in are metrics with a split transverse space, of the form

$$
\mathrm{d} s^{2}=\mathrm{e}^{2 A}\left[-4 \mathrm{~d} x^{+} \mathrm{d} x^{-}+\mathcal{H} \mathrm{d} x^{+2}\right]+\mathrm{e}^{2 B} \mathrm{~d} \vec{z}_{d}^{2}+\mathrm{e}^{2 C} \mathrm{~d} \vec{y}_{n}^{2} .
$$

The Ricci components for this metric may be obtained from (3.3) by taking $B_{i}=B$ for $i=1,2, \ldots, d$ and $B_{a}=C$ for $a=d+1, d+2, \ldots, d+n$ and furthermore by demanding that $A, B$ and $C$ are independent of $z_{a}$. The resulting Ricci components are

$$
\begin{aligned}
& R_{+-}=-g_{+-} \mathrm{e}^{-2 B}\left[\square_{d} A+\partial_{i} A \partial_{i} \mathcal{G}\right], \\
& R_{++}=-g_{++} \mathrm{e}^{-2 B}\left[\square_{d} A+\partial_{i} A \partial_{i} \mathcal{G}\right]-\frac{1}{2} \mathrm{e}^{2 A-2 B}\left[\square_{d} \mathcal{H}+\mathrm{e}^{2 B-2 C} \widetilde{\square}_{n} \mathcal{H}+\partial_{i} \mathcal{H} \partial_{i} \mathcal{G}\right], \\
& R_{a b}=-g_{a b} \mathrm{e}^{-2 B}\left[\square_{d} C+\partial_{i} C \partial_{i} \mathcal{G}\right], \\
& R_{i j}=-\partial_{i} \partial_{j} \mathcal{G}-2 \partial_{i} A \partial_{j} A+2\left(\partial_{i} A \partial_{j} B+\partial_{j} A \partial_{i} B\right)+(d-2) \partial_{i} B \partial_{j} B \\
& \quad+n\left(\partial_{i} B \partial_{j} C+\partial_{j} B \partial_{i} C-\partial_{i} C \partial_{j} C\right)-g_{i j} \mathrm{e}^{-2 B}\left[\square_{d} B+\partial_{k} B \partial_{k} \mathcal{G}\right],
\end{aligned}
$$

where $\mathcal{G}=2 A+(d-2) B+n C$ and $\square_{d} \equiv \sum_{i=1}^{d} \partial_{i} \partial_{i}$ and $\tilde{\square}_{n}=\sum_{a=d+1}^{d+n} \partial_{a} \partial_{a}$. While $\square_{d}$ is the flat transverse Laplacian, we note that the covariant Laplacian

$$
\widehat{\square}_{d} \equiv \mathrm{e}^{-2 B-\mathcal{G}} \partial_{i} \mathrm{e}^{\mathcal{G}} \partial_{i}=\mathrm{e}^{-2 B}\left[\square_{d}+\left(\partial_{i} \mathcal{G}\right) \partial_{i}\right],
$$

is in fact the natural quantity that shows up in (3.7). This is also the combination appearing in (3.4). The appearance of the transverse Laplacian is the foundation of the GarfinkleVachaspati method, which is based on finding solutions to $\square \mathcal{H}=0[15,16]$. In this sense, the present solution generating mechanism is based on finding solutions to the transverse Laplacian, but with a source on the right-hand side.

\subsection{Solution generating technique}

In this subsection, we show explicitly how the previous separation of $R_{M N}$ can be used as a solution generating technique. We briefly review some known solutions in the literature in this light, but also present new solutions. Hopefully, our presentation here will provide a unifying framework for understanding a wide class of solutions that asymptote to the BFHP solution.

The general construction states that given a supergravity solution of $S_{0}$ we can generate a solution $S_{\mathcal{H}}$ where the metric in $S_{\mathcal{H}}$ asymptotes to the BFHP solution. Let us consider the Einstein equations of motion for the seed solution $S_{0}$

$$
R_{M N}^{(0)}=T_{M N}^{(0)}
$$

This solution corresponds to $\mathcal{H}=0$. Due to the properties of the Ricci tensor we see that it is possible to construct a solution with $\mathcal{H} \neq 0$ granted we can provide a $\delta T_{M N}$ that compensates the contribution of $\mathcal{H}$ to the Ricci tensor. In particular, we see that all we need to provide is a new field whose only contribution to the stress-energy tensor is of the form $\delta T_{++}$. The candidate for such a field is a null form, naturally $F_{5}$. Note, however that this construction is not universal and one has to guarantee that the introduction of the null 5-form is compatible with the existing form fields in the seed solution. This nonuniversality forces us to consider generating solutions on a case by case basis.

3.2.1. The BFHP solution. The simplest example of an $\mathcal{H}$-deformed solution is the BFHP background itself, which can be seen as the $\mathcal{H}$-deformation of ten-dimensional Minkowski spacetime. The maximally supersymmetric plane wave solution to IIB theory was constructed 
in [6] as a supergravity background involving the metric and RR 5-form flux. In Brinkman coordinates, the solution has the form

$$
\begin{aligned}
& \mathrm{d} s^{2}=-4 \mathrm{~d} x^{+} \mathrm{d} x^{-}+\mathcal{H} \mathrm{d} x^{+2}+\mathrm{d} \vec{z}^{2} \\
& F_{5}=\mu \mathrm{d} x^{+} \wedge\left(\mathrm{d} z^{1} \wedge \mathrm{d} z^{2} \wedge \mathrm{d} z^{3} \wedge \mathrm{d} z^{4}+\mathrm{d} z^{5} \wedge \mathrm{d} z^{6} \wedge \mathrm{d} z^{7} \wedge \mathrm{d} z^{8}\right) .
\end{aligned}
$$

Having ten-dimensional Minkowski spacetime as the seed solution, its non-deformed equations of motion are trivial. For the deformed solution there is only one nontrivial equation:

$$
\square \mathcal{H}=-\mu^{2} .
$$

Here it is seen that the 5 -form serves as a source to this equation (the $\delta T_{++}$alluded to previously), resulting in a general $S O(8)$ symmetric solution [24]

$$
\mathcal{H}=c_{0}+\frac{\mathcal{Q}}{r^{6}}-\frac{1}{16} \mu^{2} r^{2} .
$$

The physical meaning of $\mathcal{Q}$ can be traced to $\mathrm{M} 2$ charge after appropriately T-dualizing and lifting the solution to $11 \mathrm{D}$ [24]. Setting $\mathcal{Q}=0$ results in the maximally supersymmetric plane wave solution.

Many important features of this solution derive from the fact that the plane wave metric admits a covariantly constant null Killing vector, $\partial / \partial x^{-}$. In particular, the only nontrivial Riemann and Ricci components are

$$
R_{+i+j}=-\frac{1}{2} \partial_{i} \partial_{j} \mathcal{H}(\vec{z}), \quad R_{++}=-\frac{1}{2} \square \mathcal{H}(\vec{z}) .
$$

It is easy to show that any generalization of the plane wave metric which preserves a covariantly constant null Killing vector does not admit an event horizon. The details of the argument were spelled out in [13].

3.2.2. $\mathcal{H}$-deformed 3-branes. We now turn to a generalization of $\mathrm{D} 3$-branes in a plane wave background. These types of backgrounds have been described from the supergravity point of view in, for example, [25-27] and from the string theoretic point of view in [28-31]. Here we view these solutions as $\mathcal{H}$-deformations of the standard D3-brane solution of IIB supergravity.

There is however an ambiguity in the form of the new 5-form needed to compensate for the introduction of non-zero $\mathcal{H}$. We begin by presenting the $\mathrm{D} 3(+,-, 1,1)$ solution. The supergravity background in question is

$$
\begin{aligned}
& \mathrm{d} s^{2}=H^{-1 / 2}\left[-4 \mathrm{~d} x^{+} \mathrm{d} x^{-}+\mathcal{H}(\vec{z}, x, y) \mathrm{d} x^{+2}+\mathrm{d} x^{2}+\mathrm{d} y^{2}\right]+H^{1 / 2} \mathrm{~d} \vec{z}_{6}^{2}, \\
& F_{5}^{(0)}=2(1+*) \mathrm{d} x^{+} \wedge \mathrm{d} x^{-} \wedge \mathrm{d} x \wedge \mathrm{d} y \wedge \mathrm{d} H^{-1}, \\
& F_{5}^{(1)}=\mu \mathrm{d} x^{+} \wedge\left(\mathrm{d} x \wedge \mathrm{d} z^{1} \wedge \mathrm{d} z^{2} \wedge \mathrm{d} z^{3}+\mathrm{d} y \wedge \mathrm{d} z^{4} \wedge \mathrm{d} z^{5} \wedge \mathrm{d} z^{6}\right),
\end{aligned}
$$

where $F_{5}^{(0)}$ corresponds to the D3-brane source (giving rise to $T_{M N}^{(0)}$ ) and $F_{5}^{(1)}$ is the compensating null 5 -form supporting the $\mathcal{H}$-deformation. We find that, as explained previously, all equations are satisfied by virtue of the seed solution with

$$
H=1+\frac{L^{4}}{r^{4}},
$$

where the six-dimensional radius is $r^{2}=\sum_{i=1}^{6} z_{i}^{2}$. We are left with only one equation involving $\mathcal{H}$ and the new source,

$$
\left[\square^{(6)}+H\left(\partial_{x}^{2}+\partial_{y}^{2}\right)\right] \mathcal{H}=-\mu^{2},
$$


with general solution of the form

$$
\mathcal{H}=c_{0}+\frac{\mathcal{Q}}{r^{4}}-\frac{1}{16} \mu^{2}\left(\rho^{2}+\frac{L^{4}}{r^{2}}\right),
$$

where $\rho^{2}=r^{2}+x^{2}+y^{2}$. For $\mathcal{Q}=0$ we recover the solution presented in [25].

Let us analyse the possibility of a horizon for this solution from the point of view of section 2. Exploring the possibility of a horizon at $r=0$, we find $a=1$ and $b=-1$. However, the behaviour of $\mathcal{H}$ (and hence the horizon analysis) depends on the charges present in the solution. For the standard D3-brane $(\mu=0, \mathcal{Q}=0)$ we find a horizon as expected. On the other hand, allowing $\mathcal{Q} \neq 0$ leads to $h=-2$. In this case, according to the criteria (2.13), $r=0$ is no longer a horizon. Finally, for $\mathcal{Q}=0$ but non-zero $\mu$, we find that the dominant term in $\mathcal{H}$, using the language of section 2, corresponds to $h<0$ and $\operatorname{sgn} \mathcal{H}=-1$ which signals a repulsive singularity. Thus the $\mathcal{H}$-deformation of D3-branes resulting in a generalization of the form $\mathrm{D} 3(+,-, 1,1)$ does not admit a regular horizon.

Another possibility of consistently introducing a null 5-form supporting non-zero $\mathcal{H}$ leads to a generalization of the $\mathrm{D} 3(+,-, 2,0)$ solution. In this case, however, in order for the null 5-form to be compatible with the initial $F_{5}^{(0)}$, the seed D3-brane must be delocalized along two of its transverse directions. The supergravity background is then of the form

$\mathrm{d} s^{2}=H^{-1 / 2}\left[-4 \mathrm{~d} x^{+} \mathrm{d} x^{-}+\mathcal{H}(\vec{z}, x, y, w, z) \mathrm{d} x^{+2}+\mathrm{d} x^{2}+\mathrm{d} y^{2}\right]+H^{1 / 2}\left[\mathrm{~d} w^{2}+\mathrm{d} z^{2}+\mathrm{d} \vec{z}_{4}^{2}\right]$,

$F_{5}^{(0)}=2(1+*) \mathrm{d} x^{+} \wedge \mathrm{d} x^{-} \wedge \mathrm{d} x \wedge \mathrm{d} y \wedge \mathrm{d} H^{-1}$,

$F_{5}^{(1)}=\mu \mathrm{d} x^{+} \wedge\left(\mathrm{d} x \wedge \mathrm{d} y \wedge \mathrm{d} w \wedge \mathrm{d} z+H \mathrm{~d} z^{1} \wedge \mathrm{d} z^{2} \wedge \mathrm{d} z^{3} \wedge \mathrm{d} z^{4}\right)$,

where again $F_{5}^{(0)}$ corresponds to the D3-brane source, while $F_{5}^{(1)}$ is now turned on corresponding to the $(+,-, 2,0)$ solution. The D3-brane is delocalized along the $v$ and $w$ directions. As a result, instead of (3.15), the seed solution has

$$
H=1+\frac{L^{2}}{r^{2}},
$$

where $r^{2}=\sum_{i=1}^{4} z_{i}^{2}$. The equation for $\mathcal{H}$ is

$$
\left[\square^{(4)}+H\left(\partial_{x}^{2}+\partial_{y}^{2}\right)+\partial_{w}^{2}+\partial_{z}^{2}\right] \mathcal{H}=-\mu^{2} H,
$$

which has a solution

$$
\mathcal{H}=c_{0}+\frac{\mathcal{Q}}{r^{2}}-\frac{1}{16} \mu^{2}\left(\rho^{2}+6 L^{2} \log r\right),
$$

where $\rho^{2}=r^{2}+x^{2}+y^{2}+w^{2}+z^{2}$. This generalizes the solution of [25]. In the notation of section 2 we find that $a=-b=1 / 2$. For $\mathcal{H}=0$ we have a horizon at $r=0$. However, for the general expression for $\mathcal{H}$ (3.21) we find $h=-1$ and subsequently the conditions for the existence of a horizon (2.13) are not satisfied. In the particular case of $\mathcal{Q}=0$ the main contribution comes from the logarithmic term in $\mathcal{H}$ which results in a regular horizon. Thus, for this type of solution we are able to successfully use the solution generating mechanism to obtain a solution which asymptotically approaches the BFHP solution and contains the same horizon as the seed solution.

3.2.3. Intersecting branes. As the above examples indicate, the $\mathcal{H}$-deformation can be easily applied to many brane solutions. For another example, we consider the intersecting D1-D5 
system. The IIB fields of interest are the metric, dilaton, RR 3-form and RR 5-form field strengths. In the Einstein frame, the appropriate equations of motion are

$$
\begin{aligned}
& R_{M N}=\frac{1}{2} \partial_{M} \phi \partial_{N} \phi+\frac{1}{96} F_{5 M N}^{2}+\frac{1}{4} \mathrm{e}^{\phi}\left(F_{3 M N}^{2}-\frac{1}{12} g_{M N} F_{3}^{2}\right), \\
& \square \phi=\frac{1}{12} \mathrm{e}^{\phi} F_{3}^{2}, \\
& \mathrm{~d} F_{3}=0, \quad d *\left(\mathrm{e}^{\phi} F_{3}\right)=0, \\
& \mathrm{~d} F_{5}=0, \quad F_{5}=* F_{5}, \quad F_{5} \wedge F_{3}=0 .
\end{aligned}
$$

As demonstrated in [25], this system admits independently both D1 $(+,-, 0,0)$ and D5 $(+,-, 2,2)$ solutions in the plane wave background. These two solutions can be combined to form a generalization of the D1-D5 solution having the form

$$
\begin{aligned}
& \mathrm{d} s^{2}=H_{1}^{-\frac{3}{4}} H_{5}^{-\frac{1}{4}}\left[-4 \mathrm{~d} x^{+} \mathrm{d} x^{-}+\mathcal{H} \mathrm{d} x^{+2}\right]+H_{1}^{\frac{1}{4}} H_{5}^{-\frac{1}{4}} \mathrm{~d} \vec{y}^{2}+H_{1}^{\frac{1}{4}} H_{5}^{\frac{3}{4}} \mathrm{~d} \vec{z}^{2}, \\
& F_{5}=\mu \mathrm{d} x^{+} \wedge\left(\mathrm{d} y^{1} \wedge \mathrm{d} y^{2} \wedge \mathrm{d} z^{1} \wedge \mathrm{d} z^{2}+\mathrm{d} y^{3} \wedge \mathrm{d} y^{4} \wedge \mathrm{d} z^{3} \wedge \mathrm{d} z^{4}\right), \\
& F_{3}=2 \mathrm{~d} x^{+} \wedge \mathrm{d} x^{-} \wedge \mathrm{d} H_{1}^{-1}+\frac{1}{6} \epsilon_{i j k l} \partial_{l} H_{5} \mathrm{~d} z^{i} \wedge \mathrm{d} z^{j} \wedge \mathrm{d} z^{k}, \\
& \mathrm{e}^{2 \phi}=H_{1} / H_{5} .
\end{aligned}
$$

Note that, for simplicity of notation, the $4+4$ split of the plane wave is in the $\left\{y^{1}, y^{2}, z^{1}, z^{2}\right\}$ and $\left\{y^{3}, y^{4}, z^{3}, z^{4}\right\}$ directions, as indicated explicitly for the 5-form. Here, $\vec{z}$ are overalltransverse coordinates, while $\vec{y}$ are relative-transverse coordinates. The D1 and D5 harmonic functions satisfy $\square H_{1}=0$ and $\square H_{5}=0$. For radial symmetry, they may be written as

$$
H_{1}=1+\frac{Q_{1}}{r^{2}}, \quad H_{5}=1+\frac{Q_{5}}{r^{2}},
$$

where $r^{2}=\sum z_{i}^{2}$ is the overall-transverse radius ${ }^{4}$.

For the plane wave background, $\mathcal{H}$ satisfies the Laplace equation with source

$$
\left[\square+H_{5} \tilde{\square}\right] \mathcal{H}=-\mu^{2} .
$$

To obtain the proper plane wave asymptotics, we take $\mathcal{H}=-\frac{1}{16} \mu^{2} \rho^{2}+\cdots$, where $\rho^{2}=\sum z_{i}^{2}+y_{i}^{2}$ contains all eight transverse coordinates. The appropriate solution to (3.25) has the form

$$
\mathcal{H}=c_{0}+\frac{Q_{w}}{r^{2}}-\frac{1}{16} \mu^{2}\left(\rho^{2}-4 Q_{5} \log r\right),
$$

where the constant $c_{0}$ may be absorbed by shifts in $x^{-}$. For $\mu^{2}=0$, this is identical to the well-known 3-charge $D=5$ black hole, lifted to ten dimensions (compare with (A.1), which is instead given in the string frame), with entropy $S \sim \sqrt{Q_{1} Q_{5} Q_{w}}$.

Let us investigate what happens to the original $r=0$ horizon of the seed solution. Here we have $a=-b=1$ and $h=-1$; this means that the second set of conditions in (2.13) for the existence of a horizon is satisfied, and we have succeeded in deforming the solution in a way that preserves the horizon of the seed solution and changes the large $|\vec{z}|$ asymptotics to that of the BFHP solution. Moreover, the horizon is non-singular, just as for the D1-D5 system in flat space.

4 As expected, by setting $\mathcal{H}$ to zero and conformally rescaling the Einstein frame metric (3.23) with a dilaton factor $\mathrm{e}^{\phi / 2}$, one reobtains the standard D1-D5 metric in the string frame, in flat space (A.1). 


\section{Non-extremal deformations}

The examples of the previous section indicate that many brane solutions initially asymptotic to Minkowski space may be given BFHP plane wave asymptotics by appropriately turning on a null 5-form. However, in all such examples, we have focused on extremal brane configurations. In this section, we turn to the possibility of constructing non-extremal deformations of the maximally symmetric BFHP solution without the introduction of additional fields. Our starting point is thus the metric and RR 5-form, initially of the form (3.10).

While the metric itself has an $S O(8)$ isometry, the true isometry group is only $S O(4) \times S O(4)$ because of the 5-form. This suggests that, when seeking non-extremal generalizations, one should be content with preserving only $S O(4) \times S O(4)$ invariance of the metric. Nevertheless, we proceed first with an ansatz that retains $S O(8)$ invariance of the metric, and subsequently turn to the $S O(4) \times S O(4)$ case.

\subsection{An SO(8) invariant deformation}

For an $S O(8)$ invariant deformation, we take as generalization of (3.10) a metric of the form

$$
\mathrm{d} s^{2}=\mathrm{e}^{2 A}\left[-4 \mathrm{~d} x^{+} \mathrm{d} x^{-}+\mathcal{H} \mathrm{d} x^{+2}\right]+\mathrm{e}^{2 B} \mathrm{~d} \vec{z}^{2},
$$

with the 5 -form unchanged. In addition to the original $\mathcal{H}(\vec{z})$, this introduces two additional 'blackening' functions $A(\vec{z})$ and $B(\vec{z})$. Since the 5-form equations of motion are trivially satisfied, we only need to be concerned with the Einstein equation. However, in this case, a non-constant function $A$ eliminates covariant constancy of $\partial / \partial x^{-}$, since $\nabla_{i}\left(\partial / \partial x^{-}\right)=$ $2 \mathrm{e}^{2 A} \Gamma^{+}{ }_{i+}=\partial_{i} \mathrm{e}^{2 A}$. Once we have relaxed the covariant constancy of $\partial / \partial x^{-}$, additional nontrivial components of the Ricci tensor show up, and there are more components of the Einstein equation to be solved. Nevertheless, as demonstrated in the previous section, the 'harmonic function' $\mathcal{H}$ only arises in the $R_{++}$component. Thus the system essentially separates into a set of source-free equations, $R_{+-}=0$ and $R_{i j}=0$, as well as an equation for $\mathcal{H}$ arising from $R_{++}$. The former equations may be solved independent of the structure of the 5 -form source. Thus we may essentially first build up a vacuum solution and then demonstrate that $\mathcal{H}$ may be consistently turned on in such a background.

The Ricci components $R_{+-}, R_{++}$and $R_{i j}$ may be read off from (3.7) for $d=8$ and $n=0$. Specializing to $S O(8)$ symmetry, we take $A$ and $B$ to be functions of $r=|\vec{z}|$ only. The resulting vacuum Einstein equations read

$$
\begin{aligned}
& A^{\prime \prime}+\frac{7}{r} A^{\prime}+2\left(A^{\prime}+3 B^{\prime}\right) A^{\prime}=0, \\
& B^{\prime \prime}+\frac{7}{r} B^{\prime}+2\left(A^{\prime}+3 B^{\prime}\right)\left(B^{\prime}+\frac{1}{r}\right)=0, \\
& A^{\prime \prime}+3 B^{\prime \prime}+A^{\prime 2}-2 A^{\prime} B^{\prime}-3 B^{\prime 2}-\frac{1}{r}\left(A^{\prime}+3 B^{\prime}\right)=0 .
\end{aligned}
$$

The first two equations are readily solved. In particular, by defining $A+3 B=\frac{1}{2} \mathcal{G}=\frac{1}{2} \log g$, we obtain the equation $\partial_{r}\left(r^{13} \partial_{r} g\right)=0$, with solution

$$
g(r)=1-\left(\frac{r_{0}}{r}\right)^{12},
$$

where we have fixed the asymptotics by demanding $g \rightarrow 1$ as $r \rightarrow \infty$. Substituting this into the first equation of (4.2), we find $\partial_{r}\left(r^{7} g \partial_{r} A\right)=0$ which admits a solution $A=\alpha \log f$ where $\alpha$ is a constant and

$$
f(r)=\frac{1-\left(r_{0} / r\right)^{6}}{1+\left(r_{0} / r\right)^{6}} .
$$


Finally, the last equation of (4.2) provides a constraint, $\alpha^{2}=7 / 16$.

Putting everything together, this yields a metric of the form

$$
\mathrm{d} s^{2}=f^{2 \alpha}\left[-4 \mathrm{~d} x^{+} \mathrm{d} x^{-}+\mathcal{H} \mathrm{d} x^{+2}\right]+f^{-\frac{2}{3} \alpha} g^{\frac{1}{3}} \mathrm{~d} \vec{z}^{2} .
$$

Until now, we have been able to completely ignore the harmonic function $\mathcal{H}(r)$. This shows up only in the $R_{++}$equation, which for (4.5) simply reads $\widehat{\square} \mathcal{H}=-\mu^{2}$, where $\widehat{\square}$ is given by (3.8), or

$$
r^{-7} \partial_{r}\left(r^{7} g \partial_{r} \mathcal{H}\right)=-\mu^{2}
$$

Just as in the equation for $A$, this is simply the transverse Laplacian, however this time with a 5 -form source. The solution is given by

$$
\mathcal{H}(r)=c_{0}-\frac{\mathcal{Q}}{2 r_{0}^{6}} \log f(r)-\frac{1}{16} \mu^{2} \int \frac{\mathrm{d}\left(r^{2}\right)}{g(r)},
$$

which is the generalization of (3.12). Even though one can solve explicitly for $\mathcal{H}$, we are mainly interested in its leading behaviour near $r_{0}$ :

$$
\mathcal{H}=-\left(\frac{\mathcal{Q}}{2 r_{0}^{6}}+\frac{\mu^{2} r_{0}^{2}}{96}\right) \log \frac{3\left(r-r_{0}\right)}{r_{0}}+\cdots, \quad r \rightarrow r_{0} .
$$

A particular case of this solution appeared in [14], where the $\mu=0$ case was considered (i.e., in the absence of 5-form flux).

The function $f$ in equation (4.4) is reminiscent of a blackening function in isotropic coordinates, and the metric (4.5) has a potential horizon at $r=r_{0}$. However, for the vacuum solution, it was shown in [14] that $r=r_{0}$ is in fact a naked curvature singularity, and not a true horizon. The addition of the 5-form flux, while yielding the proper plane wave asymptotics at infinity, $\mathcal{H}(r) \sim-\frac{1}{16} \mu^{2} r^{2}$, does not change this conclusion. This may be seen from the analysis of section 2 and in particular (2.13), where $a=\alpha \approx 0.6614$ and $b=\frac{1}{6}-\frac{1}{3} \alpha \approx-0.0538$, so that the $a-b \geqslant 1$ condition is violated. Thus we must look elsewhere for a solution with a realized horizon.

Note that this IIB background may be T-dualized and lifted to provide a solution to 11dimensional supergravity in terms of a non-extremal deformed M2-brane, which generalizes the extremal solution of [24]. The result of the lifting is

$\mathrm{d} s_{11}^{2}=\mathcal{H}^{-\frac{2}{3}} f^{-\frac{4}{3} \alpha}\left[-f^{4 \alpha} \mathrm{d} t^{2}+\mathrm{d} x_{1}^{2}+\mathrm{d} x_{2}^{2}\right]+\mathcal{H}^{\frac{1}{3}} g^{\frac{1}{3}} \mathrm{~d} \vec{z}^{2}$,

$F_{4}=\mu\left(\mathrm{d} z^{1} \wedge \mathrm{d} z^{2} \wedge \mathrm{d} z^{3} \wedge \mathrm{d} z^{4}+\mathrm{d} z^{5} \wedge \mathrm{d} z^{6} \wedge \mathrm{d} z^{7} \wedge \mathrm{d} z^{8}\right)-\mathrm{d} t \wedge \mathrm{d} x^{1} \wedge \mathrm{d} x^{2} \wedge \mathrm{d} \mathcal{H}^{-1}$,

which indicates that $\mathcal{H}$ has the form of a M2-brane harmonic function. It may be shown that this 11-dimensional solution also has a naked singularity at $r=r_{0}$.

\subsection{An $S O(4) \times S O(4)$ deformation}

Having explicitly demonstrated that the $S O(8)$ symmetric solution leads to a naked singularity, we now consider the possibility of introducing a regular horizon by deforming (3.10) in an $S O(4) \times S O(4)$ symmetric manner. This situation is very similar to that of the large black hole in $\mathrm{AdS}_{5} \times S^{5}$. We therefore proceed with a metric ansatz

$$
\mathrm{d} s^{2}=\mathrm{e}^{2 A}\left[-4 \mathrm{~d} x^{+} \mathrm{d} x^{-}+\mathcal{H} \mathrm{d} x^{+2}\right]+\mathrm{e}^{2 B} \mathrm{~d} \vec{z}^{2}+\mathrm{e}^{2 C} \mathrm{~d} \vec{y}^{2},
$$

where $\vec{z}=\left\{z^{i}\right\}, i=1,2,3,4$ and $\vec{y}=\left\{y^{a}\right\}, a=1,2,3,4$. Based on the large black hole idea, we take the functions $A(\vec{z}), B(\vec{z})$ and $C(\vec{z})$ to be functions of $\vec{z}$ only, while we allow the harmonic function $\mathcal{H}(\vec{z}, \vec{y})$ to depend on all eight transverse coordinates. The 5 -form must be 
chosen to respect both self-duality and the Bianchi identity $\mathrm{d} F_{5}=0$. For the above metric ansatz, the appropriate choice is

$F_{5}=\mu \mathrm{d} x^{+} \wedge\left(\mathrm{e}^{4 B-4 C} \mathrm{~d} z^{1} \wedge \mathrm{d} z^{2} \wedge \mathrm{d} z^{3} \wedge \mathrm{d} z^{4}+\mathrm{d} y^{1} \wedge \mathrm{d} y^{2} \wedge \mathrm{d} y^{3} \wedge \mathrm{d} y^{4}\right)$.

In fact, the combination of self-duality and Bianchi is quite restrictive, and indicates that in general we would be unable to allow $\vec{y}$ dependence in $B$ or $C$, except in the $S O(8)$ invariant case when $B=C$.

The non-vanishing components of the Ricci tensor may be obtained from (3.7) with $d=4$ and $n=4$. We again note that $\mathcal{H}$ only appears in $R_{++}$, where it enters in terms of an appropriate curved space transverse Laplacian, and that the remaining Einstein equations are simply $R_{+-}=0, R_{i j}=0$ and $R_{a b}=0$. The latter source-free equations may be solved as in the previous subsection. Defining

$$
A+B+2 C=\frac{1}{2} \mathcal{G}=\frac{1}{2} \log g, \quad A=\alpha \log f, \quad C=\beta \log f,
$$

we find

$$
g(r)=1-\left(\frac{r_{0}}{r}\right)^{4}, \quad f(r)=\frac{1-\left(r_{0} / r\right)^{2}}{1+\left(r_{0} / r\right)^{2}},
$$

where the constants $\alpha$ and $\beta$ are required to satisfy the condition

$$
\alpha^{2}+2 \alpha \beta+3 \beta^{2}=\frac{3}{8}
$$

This is the equation for an ellipse, and admits solutions for real $\alpha$ and $\beta$.

Finally, turning to the $\mathcal{H}$ equation, we find

$$
r^{-3} \partial_{r}\left(r^{3} g \partial_{r} \mathcal{H}\right)+f^{-2(\alpha+3 \beta)} g^{2} \tilde{\square} \mathcal{H}=-\mu^{2} f^{-4(\alpha+3 \beta)} g^{2} .
$$

Unlike in the $S O(8)$ invariant case, (4.6), here the source term is no longer simply constant. In addition, while $\mathcal{H}$ is a function of both $r$ and $\tilde{r} \equiv|\vec{y}|$, these two transverse coordinates enter asymmetrically in the above. However, this equation may be solved by assuming that $\mathcal{H}$ separates as a sum of functions of $r$ and $\tilde{r}$ respectively. Although other solutions are possible, we simply choose $\mathcal{H}=-\frac{1}{16} \mu^{2}\left(r^{2}+\tilde{r}^{2}\right)+\overline{\mathcal{H}}(r)$ so as to reproduce the asymptotics at infinity. Then equation (4.15) becomes

$$
r^{-3} \partial_{r}\left(r^{3} g \partial_{r} \overline{\mathcal{H}}\right)=-\frac{1}{2} \mu^{2} f^{-2(\alpha+3 \beta)} g^{2}\left(2 f^{-2(\alpha+3 \beta)}-1\right)
$$

which may be integrated twice to obtain $\overline{\mathcal{H}}(r)$. The large distance asymptotics has the form

$$
\mathcal{H}=c_{0}+\frac{\mathcal{Q}}{r^{2}}-\frac{1}{16} \mu^{2}\left(r^{2}+\tilde{r}^{2}\right)-3(\alpha+3 \beta) \mu^{2} r_{0}^{2} \log \frac{r}{r_{0}}+\cdots,
$$

while the behaviour near $r_{0}$ is either logarithmic for $\alpha+3 \beta \leqslant \frac{3}{4}$ or given by a power law, $\mathcal{H} \sim\left(r-r_{0}\right)^{-[4(\alpha+3 \beta)-3]}$, otherwise.

In this manner, we have obtained a $S O(4) \times S O(4)$ invariant deformation of the maximally symmetric plane wave of the form

$$
\begin{aligned}
& \mathrm{d} s^{2}=f^{2 \alpha}\left[-4 \mathrm{~d} x^{+} \mathrm{d} x^{-}+\mathcal{H} \mathrm{d} x^{+2}\right]+f^{-2(\alpha+2 \beta)} g \mathrm{~d} \vec{z}^{2}+f^{2 \beta} \mathrm{d} \vec{y}^{2}, \\
& F_{5}=\mu \mathrm{d} x^{+} \wedge\left(f^{-4(\alpha+3 \beta)} g^{2} \mathrm{~d} z^{1} \wedge \mathrm{d} z^{2} \wedge \mathrm{d} z^{3} \wedge \mathrm{d} z^{4}+\mathrm{d} y^{1} \wedge \mathrm{d} y^{2} \wedge \mathrm{d} y^{3} \wedge \mathrm{d} y^{4}\right) .
\end{aligned}
$$

This solution is similar to the $S O(8)$ invariant example of (4.5), in that the harmonic function solving (4.16) almost entirely decouples from the Einstein equations. This is a feature of retaining $\partial / \partial x^{-}$as a null Killing direction, and ensures that the blackening functions $f$ and $g$ essentially have the form they would for a vacuum solution, even in the presence of the 5 -form flux. 
We now investigate whether this solution admits a horizon at $r=r_{0}$. Since both $f \sim r-r_{0}$ and $g \sim r-r_{0}$ near $r_{0}$, the leading expansion of the warp factors is

$$
\mathrm{e}^{2 A} \sim\left(r-r_{0}\right)^{2 \alpha}, \quad \mathrm{e}^{2 B} \sim\left(r-r_{0}\right)^{1-2(\alpha+2 \beta)} .
$$

This yields $a=\alpha$ and $b=\frac{1}{2}-\alpha-2 \beta$. For $\alpha+3 \beta \leqslant \frac{3}{4}$, so that $\mathcal{H}$ is logarithmic near $r_{0}$, the criteria for a horizon, (2.13), are then

$$
\beta<\frac{3}{4}, \quad \alpha+\beta \geqslant \frac{3}{4} .
$$

The second inequality is incompatible with the ellipse condition, (4.14), since

$$
\alpha^{2}+2 \alpha \beta+3 \beta^{2}-\frac{3}{8}=(\alpha+\beta)^{2}+2 \beta^{2}-\frac{3}{8} \geqslant 2 \beta^{2}+\frac{3}{16}>0 \text {. }
$$

On the other hand, if $\mathcal{H}$ diverges as a power law, the existence of a horizon requires $\alpha+\beta \geqslant \frac{3}{4}+\frac{|h|}{2}$, which is also incompatible with the ellipse condition, since it amounts to $\alpha^{2}+2 \alpha \beta+3 \beta^{2}-\frac{3}{8} \geqslant\left(\frac{3}{4}+\frac{|h|}{2}\right)^{2}+2 \beta^{2}-\frac{3}{8}>0$. Thus all pairs $(\alpha, \beta)$ on the ellipse (4.14) parametrize solutions that have naked singularities at $r=r_{0}$.

\section{No horizons for metrics admitting a null Killing vector}

Based on the observations made in the previous section, one may develop the suspicion that the class of static metrics preserving a null Killing vector do not allow for a regular horizon. Here we demonstrate that this is indeed the case by proving a no-go theorem. More precisely, we shall prove that

Theorem 1. In the presence of matter contributing only to $T_{++}$, and with $\partial / \partial x^{+}$an asymptotically timelike Killing vector, the following metric

$$
\mathrm{d} s^{2}=\mathrm{e}^{2 A(r)}\left[-4 \mathrm{~d} x^{+} \mathrm{d} x^{-}+\mathcal{H} \mathrm{d} x^{+2}\right]+\mathrm{e}^{2 B(r)}\left[\mathrm{d} r^{2}+r^{2} \mathrm{~d} \Omega_{n}^{2}\right]+\sum_{i} \mathrm{e}^{2 C_{i}(r)} \mathrm{d} y_{i}^{2}
$$

cannot admit a regular $S O(n+1)$ invariant horizon

Before proceeding to the proof, let us discuss the conditions of the theorem. The fact that we consider only null matter puts us in the simplest class of metrics that asymptote to the BFHP solution with no other fields turned on. Taking $x^{+}$as an asymptotic time coordinate (corresponding to $\partial / \partial x^{+}$being a timelike Killing vector) is a natural consequence of the requirement that the metric asymptotes to the BFHP solution $\left(\mathcal{H} \sim-\frac{1}{16} \mu^{2} \rho^{2}\right)$. We will comment on the consequences of relaxing these conditions at the end of this section.

The proof of the no-go theorem relies on the separation property of the metric in the presence of a null Killing vector, (1.3). This separation implies that the vacuum Einstein equations for (5.1) are equivalent to those of a 'conventional' $\mathcal{H}=0$ metric supplemented by an equation on $\mathcal{H}$ (resulting from the $R_{++}$equation). Thus we begin by considering a metric, not in light-cone variables, but rather written as

$$
\mathrm{d} s^{2}=-\mathrm{e}^{2 A(r)} \mathrm{d} t^{2}+\mathrm{e}^{2 B(r)}\left[\mathrm{d} r^{2}+r^{2} \mathrm{~d} \Omega_{n}^{2}\right]+\sum_{a} \mathrm{e}^{2 C_{a}(r)} \mathrm{d} y_{a}^{2} .
$$

The radially symmetric vacuum Einstein equations are (no sums except where explicit)

$$
\begin{aligned}
& 0=R_{t t}=\mathrm{e}^{2 A-2 B}\left[A^{\prime \prime}+\frac{n}{r} A^{\prime}+A^{\prime}\left(A^{\prime}+(n-1) B^{\prime}+\sum C_{a}^{\prime}\right)\right], \\
& 0=R_{a a}=-\mathrm{e}^{2 C_{a}-2 B}\left[C_{a}^{\prime \prime}+\frac{n}{r} C_{a}^{\prime}+C_{a}^{\prime}\left(A^{\prime}+(n-1) B^{\prime}+\sum C_{j}^{\prime}\right)\right], \\
& 0=R_{\alpha \beta}=-\mathrm{e}^{-2 B} g_{\alpha \beta}\left[B^{\prime \prime}+\frac{n}{r} B^{\prime}+\left(B^{\prime}+\frac{1}{r}\right)\left(A^{\prime}+(n-1) B^{\prime}+\sum C_{a}^{\prime}\right)\right], \\
& 0=R_{r r}=-A^{\prime \prime}-n B^{\prime \prime}-\sum C_{a}^{\prime \prime}-A^{\prime 2}-\sum C_{a}^{\prime 2}+B^{\prime}\left(A^{\prime}+\sum C_{a}^{\prime}-\frac{n}{r}\right),
\end{aligned}
$$


where $\alpha$ and $\beta$ denote directions on $S^{n}$. As before, the first three equations admit a solution of the form

$$
A+(n-1) B+\sum C_{a}=\mathcal{G}=\log g, \quad A=\alpha \log f, \quad C_{a}=\gamma_{a} \log f,
$$

where

$$
g(r)=1-\left(\frac{r_{0}}{r}\right)^{2(n-1)}, \quad f(r)=\frac{1-\left(r_{0} / r\right)^{n-1}}{1+\left(r_{0} / r\right)^{n-1}} .
$$

We stress that this is the most general solution consistent with the natural boundary conditions at infinity.

Making use of the identity

$$
\frac{g^{\prime}}{g}\left(\frac{g^{\prime}}{g}+\frac{2(n-1)}{r}\right)=\left(\frac{f^{\prime}}{f}\right)^{2}
$$

we find that the $R_{r r}$ equation yields the algebraic constraint

$$
(n-1)\left(\alpha^{2}+\sum \gamma_{a}^{2}\right)+\left(\alpha+\sum \gamma_{a}\right)^{2}=n
$$

Note that all parameters $\left\{\alpha, \gamma_{a}\right\}$ enter symmetrically in the constraint. This indicates that singling out the time coordinate in (5.1) has no effect at the level of the solution. On the other hand, since we are interested in identifying a potential horizon, we find it useful to maintain this $\left\{\alpha, \gamma_{a}\right\}$ split.

Any set of parameters satisfying (5.7) provides a solution to the vacuum Einstein equations. However, we must further examine whether the solution admits a regular horizon. To do so, we consider timelike radial geodesics in the background (5.2). Since the metric is time independent, we immediately identify a conserved energy $E=\mathrm{e}^{2 A} \dot{t}$, where dots refer to derivatives with respect to the proper time, $\tau$. Radial geodesics are then parametrized by solutions of

$$
\dot{r}^{2}=\mathrm{e}^{-2 B}\left(\mathrm{e}^{-2 A} E^{2}-1\right)
$$

Based on the behaviour of $f$ and $g$ given in (5.5), we may expand $\mathrm{e}^{2 A}$ and $\mathrm{e}^{2 B}$ near $r_{0}$. For $\mathrm{e}^{2 A} \rightarrow 0$, the first term dominates, and we find

$$
\mathrm{d} \tau \sim E^{-1}\left(r-r_{0}\right)^{\alpha-\frac{1}{n-1}\left(\alpha+\sum \gamma_{a}-1\right)} \mathrm{d} r,
$$

or

$$
\mathrm{d} t \sim\left(r-r_{0}\right)^{-\alpha-\frac{1}{n-1}\left(\alpha+\sum \gamma_{a}-1\right)} \mathrm{d} r .
$$

For $r=r_{0}$ to be a horizon, we demand that the exponent in (5.10) is such that the geodesic cannot reach $r_{0}$ in finite coordinate time $t$. This gives rise to the condition

$$
\alpha+\frac{1}{n} \sum \gamma_{a} \geqslant 1
$$

for the existence of a horizon.

To determine whether solutions to the constraint (5.7) are compatible with the horizon condition, we rewrite (5.7) as

$$
\left(\alpha+\frac{1}{n} \sum \gamma_{a}\right)^{2}+\frac{n-1}{n^{2}}\left(n \sum \gamma_{a}^{2}+\left(\sum \gamma_{a}\right)^{2}\right)=1
$$

However, since the first term is bounded below by unity from (5.11), and since the remaining terms are sums of squares, we see that the only satisfactory solution has $\alpha=1$ and all $\gamma_{a}$ set to zero. In this case, the metric (5.2) is especially simple, and has the form

$$
\mathrm{d} s^{2}=-\left(\frac{1-\left(r_{0} / r\right)^{n-1}}{1+\left(r_{0} / r\right)^{n-1}}\right)^{2} \mathrm{~d} t^{2}+\left(1+\left(r_{0} / r\right)^{n-1}\right)^{\frac{2}{n-1}}\left[\mathrm{~d} r^{2}+r^{2} \mathrm{~d} \Omega_{n}^{2}\right]+\mathrm{d} \vec{y}^{2} \text {. }
$$


This is simply an $(n+2)$-dimensional Schwarzschild black hole (written in isotropic coordinates) tensored with additional flat dimensions.

Thus we have shown that metrics of the form (5.2) only admit $S O(n+1)$ invariant horizons of the Schwarzschild form, (5.13). This is perhaps not very surprising, as it corresponds to some limited form of a uniqueness theorem for black holes ${ }^{5}$. Before returning to the light-cone metric, (5.1), we note that black $p$-branes of the general form [32]

$$
\mathrm{d} s^{2}=\mathrm{e}^{2 A}\left[-f \mathrm{~d} t^{2}+\mathrm{d} \vec{y}^{2}\right]+\mathrm{e}^{2 B}\left[f^{-1} \mathrm{~d} r^{2}+r^{2} \mathrm{~d} \Omega_{n}^{2}\right]
$$

fall into this category of blackening, and indeed have regular horizons.

Finally, we are in a position to make contact with the generalized pp-wave metric of (5.1). As demonstrated in section 3, because of the null Killing vector $\partial / \partial x^{-}$, the vacuum Einstein equations for (5.1) are identical to that of (5.2) with an additional longitudinal coordinate (say $y^{0}$ ) satisfying the condition $\gamma_{0}=\alpha$, supplemented with a transverse Laplace equation on $\mathcal{H}$

$$
\left(f^{\frac{2}{n-1}\left(\alpha+\sum_{a \geqslant 0} \gamma_{a}\right)} g^{-\frac{2}{n-1}}\right) r^{-n} g^{-1} \partial_{r}\left(r^{n} g \partial_{r} \mathcal{H}\right)+\sum_{b \geqslant 1} f^{-2 \gamma_{b}} \partial_{b}^{2} \mathcal{H}=-2 f^{-2 \alpha} T_{++}
$$

(where the Einstein equation is $R_{++}=T_{++}$). This has no effect on the blackening functions $f$ and $g$ of (5.5).

Assuming $\partial / \partial x^{+}$to be asymptotically timelike, we now examine the conditions, (2.13), which determine the existence of an event horizon for the generalized pp-wave metric (5.1). Since these conditions depend on the form of $\mathcal{H}$, we consider several cases in turn. First, we assume that $\mathcal{H}$ is logarithmic or finite near the horizon. Then the horizon conditions are simply

$$
\alpha+\beta>-1, \quad \alpha-\beta \geqslant 1,
$$

where $\beta=\left(1-\alpha-\sum_{a \geqslant 0} \gamma_{a}\right) /(n-1)$, in accord with previous calculations. Note that the second inequality is the same condition as (5.11), which determined the horizon for the metric (5.2), except that for the metric (5.1) we have $\gamma_{0}=\alpha$. Recalling that the $r r$ Einstein equation imposes a constraint (5.7) which is compatible with the conditions defining an event horizon only if $\gamma_{a}=0$ for all $a$, we see that we end up with a contradiction. Next, we consider the case where $\mathcal{H}$ grows near the horizon $(h<0)$; this corresponds to the second case of $(2.13)$. However, now the second horizon condition is of the form $\alpha-\beta \geqslant 1+|h|>1$ (since $|h|>0$ ), which is even stronger than (5.16), and hence is never satisfied. Finally, we note that if $\mathcal{H}$ blows up negative near $r_{0}$ (either logarithmically or as a power) we end up with a repulsive singularity.

As a result, we have demonstrated that all solutions of the form (5.1) with null matter are necessarily naked singularities (except for the trivial Minkowski vacuum). The essential feature here is that, to maintain a null Killing vector, the 'light-cone' directions $x^{+}$and $x^{-}$ must be 'blackened' simultaneously. This would be similar to seeking a black string solution of the form

$$
\mathrm{d} s^{2}=\mathrm{e}^{2 A} f\left[-\mathrm{d} t^{2}+\mathrm{d} z^{2}\right]+\mathrm{e}^{2 B}\left[f^{-1} \mathrm{~d} r^{2}+r^{2} \mathrm{~d} \Omega_{n}^{2}\right],
$$

which does not admit regular solutions, in contrast to the appropriate blackening ansatz of the form (5.14). As a result, to obtain a reasonable horizon for the vacuum solution, we must relax the null Killing vector condition (at least near the horizon).

We now comment on how the theorem may be modified if any of the conditions is relaxed. Firstly, the introduction of additional non-null matter would modify the Einstein equations

5 Of course, consideration of black hole uniqueness is considerably different in dimensions greater than four. Here we are not claiming to make a general statement, but rather a statement only in the context of vacuum solutions of the form (5.2). 
that determine $A, B$ and $C_{i}$ in a way that depends on the concrete type of matter involved. Thus we are unable to make any general statements without explicitly specifying the non-null matter content. As indicated in the next section, turning on additional matter is not always enough for the evasion of the no-go theorem.

Secondly, the identification of an asymptotic time coordinate is another crucial condition. So long as $\mathcal{H}<0$ in the asymptotic region, $\partial / \partial x^{+}$is asymptotically a timelike Killing vector, and the above analysis applies. However, for $\partial / \partial x^{+}$null or spacelike, one must instead resort to the horizon criteria (2.14). For $h \geqslant 0$ the condition is precisely what we had previously, (5.16), and the no-go theorem can be repeated along the same lines. On the other hand, for $h<0$ the horizon condition becomes $\alpha-\beta \geqslant 1-|h|$. In particular, it is a weaker condition than that used in proving the no-go theorem. In this sense, provided $\partial / \partial x^{+}$is not timelike, turning on $\mathcal{H}$ improves the chances of having a regular event horizon. As indicated by the examples considered in section 2 , solutions are easily found that have a regular horizon.

Finally, it is interesting to note that generically the equation for $\mathcal{H}$ contains a generalized Laplacian that allows for a homogeneous part in the solution of $\mathcal{H}$. Namely, there is usually the possibility of adding a term such that $\widehat{\square}_{d} \mathcal{H}=0$. The solution to this part contains, in principle, $h<0$ and therefore creates room for having an event horizon whenever the asymptotic time has a linear component along $x^{-}$.

\section{Turning on additional sources}

Since deformations of the plane wave background with any splitting and global symmetry $S O(m) \times S O(n) \times S O(p) \times \cdots$ fall into the category of ansätze dealt with by the preceding no-go theorem, one might hope that turning on additional fluxes (i.e., matter) would help in evading the previous negative outcome. Perhaps the most natural starting point is a string-type deformation of the plane wave, i.e., an object extended in the $(+,-)$ directions. However, based on the general structure of the Einstein equations, it is also likely that any D-brane solution would admit a non-extremal deformation. Thus, after considering the string-like case, we turn to ansätze which generalize the extremal D-brane solutions in the maximal supersymmetric plane wave background of the type IIB supergravity.

\subsection{Sourcing a string}

The string ansatz which we analyse has a partial smearing in two transverse directions, with the metric ansatz corresponding to a $2+6$ split

$\mathrm{d} s^{2}=\mathrm{e}^{2 A(r)}\left[-4 \mathrm{~d} x^{+} \mathrm{d} x^{-}+\mathcal{H} \mathrm{d} x^{+2}\right]+\mathrm{e}^{2 B(r)}\left[\mathrm{d} r^{2}+r^{2} \mathrm{~d} \Omega_{5}^{2}\right]+\mathrm{e}^{2 C(r)}\left(\mathrm{d} y_{1}^{2}+\mathrm{d} y_{2}^{2}\right)$,

and with the NS-NS 2-form and dilaton field given by

$$
H_{+-r}=F^{\prime}(r), \quad \square \phi=-\frac{1}{12} \mathrm{e}^{-\phi} H^{2} .
$$

The stress tensor components determined by these fields are

$$
\begin{aligned}
& T_{+-}=\frac{3}{16} \mathrm{e}^{-2 A-2 B} \mathrm{e}^{-\phi} F^{\prime}(r)^{2}, \\
& T_{a b}=\frac{1}{32} \delta_{a b} \mathrm{e}^{-4 A-2 B+2 C} \mathrm{e}^{-\phi} F^{\prime}(r)^{2}, \\
& T_{i j}=\frac{1}{32} \mathrm{e}^{-4 A} \mathrm{e}^{-\phi} F^{\prime}(r)^{2}\left(\delta_{i j}-4 \hat{x}_{i} \hat{x}_{j}\right)+\frac{1}{2} \partial_{i} \phi \partial_{j} \phi .
\end{aligned}
$$

So instead of solving for vacuum Einstein equations for all components with the notable exception of the ++ component, we now have sources for each Einstein equation. However, in 
taking appropriate linear combinations of the $(+-),(a b)$ and the $i j$ components, the sources cancel and we can solve for

$$
A+C+2 B=\frac{1}{2} \mathcal{G}=\frac{1}{2} \log g, \quad A+3 C=\gamma \log f,
$$

where $f, g$ are

$$
g(r)=1-\left(\frac{r_{0}}{r}\right)^{8}, \quad f(r)=\frac{1-\left(r_{0} / r\right)^{4}}{1+\left(r_{0} / r\right)^{4}},
$$

and $\gamma$ is a constant of integration.

To proceed, we note that the NS-NS and dilaton field equations yield

$$
F^{\prime}=-8 \mathrm{e}^{\phi+4 A} \frac{L^{4}}{r^{5} g}, \quad \phi^{\prime \prime}+\phi^{\prime}\left(\frac{g^{\prime}}{g}+\frac{5}{r}\right)=8 \mathrm{e}^{\phi+4 A} \frac{L^{8}}{r^{10} g^{2}},
$$

where $L$ is an integration constant related to the NS-NS 3-form (string) charge. Finally, the remaining Einstein equations read

$$
\begin{gathered}
A^{\prime \prime}+A^{\prime}\left(\frac{g^{\prime}}{g}+\frac{5}{r}\right)=6 \mathrm{e}^{\phi+4 A} \frac{L^{8}}{r^{10} g^{2}}, \\
A^{\prime \prime}+C^{\prime \prime}+2 B^{\prime \prime}-\frac{1}{r}\left(A^{\prime}+C^{\prime}+2 B^{\prime}\right)+A^{\prime 2}+C^{\prime 2}-2 B^{\prime 2}-2 A^{\prime} B^{\prime}-2 B^{\prime} C^{\prime} \\
=-\frac{1}{4} \phi^{\prime 2}+4 \mathrm{e}^{\phi+4 A} \frac{L^{8}}{r^{10} g^{2}} .
\end{gathered}
$$

Combining the first equation of (6.7) with the second equation of (6.6) allows us to solve for $\phi$ in terms of $A$. We obtain

$$
\frac{\phi}{4}-\frac{A}{3}=\beta \log f
$$

where $\beta$ is an (as yet) undetermined constant. Now, using (6.4) and (6.8), as well as the identity

$$
\left(\frac{g^{\prime}}{g}\right)^{2}+\frac{8}{r} \frac{g^{\prime}}{g}=\left(\frac{f^{\prime}}{f}\right)^{2}
$$

the second equation of (6.7) can be rewritten as an equation for $A$ only,

$$
\left[\left(\mathrm{e}^{-8 \hat{A} / 3}\right)^{\prime}\right]^{2}-\lambda^{2}\left(\mathrm{e}^{-8 \hat{A} / 3}\right)^{2}\left(\frac{f^{\prime}}{f}\right)^{2}=16 \frac{L^{8}}{r^{10} g^{2}},
$$

where

$$
\hat{A}=A+\frac{3 \beta}{4} \log f
$$

and

$$
\lambda^{2}=\frac{15-4 \gamma^{2}-72 \beta^{2}}{6} .
$$

This equation admits a solution

$$
\mathrm{e}^{-8 \hat{A} / 3}=H \equiv \frac{L^{4}}{2 \lambda r_{0}^{4}} \sinh (c-\lambda \log f)
$$

where $c$ is an integration constant (chosen so that $H \rightarrow 1$ as $r \rightarrow \infty$ ) and we assume that $\lambda^{2}$ is positive. 
Putting the above results together, we find the string solution to have the form

$$
\begin{gathered}
\mathrm{d} s^{2}=H^{-\frac{3}{4}} f^{-\frac{3}{2} \beta}\left[-4 \mathrm{~d} x^{+} \mathrm{d} x^{-}+\mathcal{H} \mathrm{d} x^{+2}\right] \\
+H^{\frac{1}{4}} f^{\frac{1}{2} \beta-\frac{1}{3} \gamma}\left[g^{\frac{1}{2}}\left(\mathrm{~d} r^{2}+r^{2} \mathrm{~d} \Omega_{5}^{2}\right)+f^{\gamma}\left(\mathrm{d} y_{1}^{2}+\mathrm{d} y_{2}^{2}\right)\right], \\
H_{+-r}=-8 H^{-2} \frac{L^{4}}{r^{5} g}, \quad \mathrm{e}^{2 \phi}=H^{-1} f^{6 \beta} .
\end{gathered}
$$

Here $H$, defined in (6.13), is identified with the fundamental string, since $H \approx 1+L^{4} / r^{4}$ in the limit $r_{0} \rightarrow 0$. Note, however, that $H$ is modified from the standard F1 harmonic function for finite $r_{0}$, and develops a singularity:

$$
H \sim\left(r-r_{0}\right)^{-|\lambda|} \text { as } r \rightarrow r_{0} .
$$

As a result, the behaviour of the metric near $r_{0}$ is given by

$$
\mathrm{e}^{2 A} \sim\left(r-r_{0}\right)^{\frac{3}{4}|\lambda|-\frac{3}{2} \beta}, \quad \mathrm{e}^{2 B} \sim\left(r-r_{0}\right)^{-\frac{1}{4}|\lambda|+\frac{1}{2} \beta-\frac{1}{3} \gamma+\frac{1}{2}} .
$$

In order to investigate the horizon properties of this solution, we need one extra piece of information, namely the behaviour of $\mathcal{H}$. From the $(++)$ Einstein equation we obtain

$$
\widehat{\square} \mathcal{H}+\mathrm{e}^{-2 C} \tilde{\square} \mathcal{H}=-\mu^{2} \mathrm{e}^{-2 A-6 B-2 C},
$$

where $\widehat{\square}$ is defined in (3.8) and $\tilde{\square}=\partial_{y_{1}}^{2}+\partial_{y_{2}}^{2}$. Again, writing $\mathcal{H}=-\frac{1}{16} \mu^{2}\left(r^{2}+y_{1}^{2}+y_{2}^{2}\right)+\overline{\mathcal{H}}(r)$ to guarantee proper asymptotic behaviour, the above equation becomes

$$
r^{-5} \partial_{r}\left(r^{5} g \partial_{r} \overline{\mathcal{H}}\right)=\frac{1}{4} \mu^{2}\left(g^{\frac{3}{2}} f^{-\gamma}-g\right) .
$$

In general, solutions to (6.18) will develop a logarithmic singularity at $r_{0}$ (so long as $\gamma<\frac{5}{2}$, which is always satisfied for $\lambda^{2}$ positive). Thus, from (2.13), the criteria for the existence of a regular horizon are

$$
|\lambda|>2 \beta+\frac{2}{3} \gamma-5, \quad|\lambda| \geqslant 2 \beta-\frac{1}{3} \gamma+\frac{5}{2},
$$

where $\lambda$ is given in (6.12). It is easy to see that, while the first inequality is always satisfied, the second one is never satisfied for real $\beta$ and $\gamma$. Thus we must conclude that this solution is ill behaved whenever $r_{0} \neq 0$. In fact, $r=r_{0}$ is a naked singularity, as the curvature invariant $R_{\mu \nu \rho \sigma} R^{\mu \nu \rho \sigma}$ blows up at $r_{0}$.

\subsection{Deforming the extremal D-branes}

In section 3.2.2, we have examined extremal D3-branes in a plane wave background. Here we consider non-extremal deformations of both the D3 $(+,-, 1,1)$ and D3 $(+,-, 2,0)$ branes. Even though some of these solutions were naked singularities one might hope that a non-zero Hawking temperature horizon cloaks the original singularity as was the case for the non-zero temperature generalization of the Klebanov-Tseytlin solution [33]. Of course, the separation property of the Ricci tensor (1.3) into background and plane wave pieces allows non-extremal deformations of arbitrary D-branes as well. However, we are content with presenting the following examples.

6.2.1. The $\mathrm{D} 3(+,-, 1,1)$ branes. For the $\mathrm{D} 3(+,-, 1,1)$ brane, the supergravity background in question is given in (3.14). A natural generalization of this solution is given by

$$
\begin{aligned}
& \mathrm{d} s^{2}=\mathrm{e}^{2 A}\left[-4 \mathrm{~d} x^{+} \mathrm{d} x^{-}+\mathcal{H} \mathrm{d} x^{+2}\right]+\mathrm{e}^{2 B} \mathrm{~d} \vec{z}_{6}^{2}+\mathrm{e}^{2 C}\left(\mathrm{~d} x^{2}+\mathrm{d} y^{2}\right), \\
& F_{5}^{(0)}=2(1+*) \mathrm{d} x^{+} \wedge \mathrm{d} x^{-} \wedge \mathrm{d} x \wedge \mathrm{d} y \wedge \mathrm{d} \mathrm{e}^{2 D} \\
& F_{5}^{(1)}=\mu \mathrm{d} x^{+} \wedge\left(\mathrm{d} x \wedge \mathrm{d} z^{1} \wedge \mathrm{d} z^{2} \wedge \mathrm{d} z^{3}+\mathrm{d} y \wedge \mathrm{d} z^{4} \wedge \mathrm{d} z^{5} \wedge \mathrm{d} z^{6}\right) .
\end{aligned}
$$


This allows for both additional blackening functions in the metric and a possible charge modification in the 5-form. The energy-momentum tensor for this configuration, $T_{M N}=$ $\frac{1}{96} F_{M N}^{2}$, decomposes as a sum of two independent components, $T_{M N}=T_{M N}^{(0)}+T_{M N}^{(1)}$, where

$$
\begin{aligned}
& T_{\mu \nu}^{(0)}=-\frac{1}{4} g_{\mu \nu} \mathrm{e}^{-4 A-2 B-4 C+4 D}\left(\partial_{i} D\right)^{2} \quad(\mu, v=+,-, x, y), \\
& T_{i j}^{(0)}=\frac{1}{4} \mathrm{e}^{-4 A-4 C+4 D}\left[\delta_{i j}\left(\partial_{k} D\right)^{2}-2 \partial_{i} D \partial_{j} D\right],
\end{aligned}
$$

is a standard D3-brane source, while

$$
T_{++}^{(1)}=\frac{1}{2} \mu^{2} \mathrm{e}^{-6 B-2 C}
$$

corresponds to the plane wave deformation. The vanishing of a possible cross-term between $F_{5}^{(0)}$ and $F_{5}^{(1)}$ is not a priori guaranteed, but is crucial for the existence of the solution [25].

Since the Ricci tensor of (3.4) similarly splits into two components, the equations of motion separate into: (i) the standard 3-brane equations $R_{M N}^{(0)}=T_{M N}^{(0)}$ (for functions $A, B, C$ and $D$ ) and (ii) a Poisson equation for $\mathcal{H}$

$$
\widehat{\square} \mathcal{H}+\mathrm{e}^{-2 C} \tilde{\square} \mathcal{H}=-\mu^{2} \mathrm{e}^{-2 A-6 B-2 C},
$$

where $\widehat{\square}$ is defined in (3.8) and $\tilde{\square}=\partial_{x}^{2}+\partial_{y}^{2}$. Note that this is identical to (6.17), which defined $\mathcal{H}$ for the $\mathrm{F} 1$ string solution. This is expected, of course, since $\mathcal{H}$ characterizes the plane wave part of the metric, and obeys an equation sensitive only to the splitting of the transverse directions, in this case $2+6$.

From the standard 3-brane equations, we can easily obtain certain linear combinations of the warp factors in terms of the blackening functions $f(r)$ and $g(r)$ :

$$
\begin{aligned}
& A+2 B+C=\frac{1}{2} \mathcal{G}=\frac{1}{2} \log g, \quad A-C=\gamma \log f, \\
& g(r)=1-\left(\frac{r_{0}}{r}\right)^{8}, \quad f(r)=\frac{1-\left(r_{0} / r\right)^{4}}{1+\left(r_{0} / r\right)^{4}} .
\end{aligned}
$$

Furthermore, the only nontrivial Bianchi identity

$$
\left(r^{5} \mathrm{e}^{-2 A+4 B-2 C}\left(\mathrm{e}^{2 D}\right)^{\prime}\right)^{\prime}=0
$$

can be integrated once to yield the D3-brane potential in terms of the other warp factors. Finally, just as in the string solution, the remaining Einstein equation can be written, after a little algebra, as

$$
\left[\left(\mathrm{e}^{-4 \hat{A}}\right)^{\prime}\right]^{2}-\lambda^{2}\left(\mathrm{e}^{-4 \hat{A}}\right)^{2}\left(\frac{f^{\prime}}{f}\right)^{2}=16 \frac{L^{8}}{r^{10} g^{2}},
$$

where

$$
\hat{A}=A-\frac{\gamma}{2} \log f
$$

and

$$
\lambda^{2}=\frac{5-4 \gamma^{2}}{2} .
$$

This equation admits a solution of the form

$$
\mathrm{e}^{-4 \hat{A}}=H \equiv \frac{L^{4}}{2 \lambda r_{0}^{4}} \sinh (c-\lambda \log f)
$$

where $c$ is an integration constant, chosen so that $H \rightarrow 1$ as $r \rightarrow \infty$. The D3 $(+,-, 1,1)$ metric is thus given by

$\mathrm{d} s^{2}=H^{-\frac{1}{2}} f^{\gamma}\left[-4 \mathrm{~d} x^{+} \mathrm{d} x^{-}+\mathcal{H} \mathrm{d} x^{+2}+f^{-2 \gamma}\left(\mathrm{d} x^{2}+\mathrm{d} y^{2}\right)\right]+H^{\frac{1}{2}} g^{\frac{1}{2}}\left[\mathrm{~d} r^{2}+r^{2} \mathrm{~d} \Omega_{5}^{2}\right]$. 
As expected, our solution reduces to the extremal case of (3.14) if we set $r_{0}=0$ (i.e., $f=g=1$ ) [25]. Note that, in this extremal limit, $H=1+L^{4} / r^{4}$ is simply the D 3 harmonic function, while for finite $r_{0}$, we have instead $H \sim\left(r-r_{0}\right)^{-|\lambda|}$ as $r \rightarrow r_{0}$.

The horizon analysis then proceeds as in the string case. By writing $\mathcal{H}=-\frac{1}{16} \mu^{2}\left(r^{2}+\right.$ $\left.x^{2}+y^{2}\right)+\overline{\mathcal{H}}(r)$, we obtain

$$
r^{-5} \partial_{r}\left(r^{5} g \partial_{r} \overline{\mathcal{H}}\right)=\frac{1}{4} \mu^{2}\left(g^{\frac{3}{2}} f^{\gamma} H-g\right),
$$

which develops at most a logarithmic singularity at $r_{0}$. As a result, the conditions for a regular horizon are

$$
\gamma>-\frac{5}{2}, \quad|\lambda|+\gamma \geqslant \frac{5}{2} .
$$

Again, the second inequality is never solved, and this solution corresponds to a naked singularity.

6.2.2. The D3 $(+,-, 2,0)$ branes. The D3 $(+,-, 2,0)$ branes of $(3.18)$ span the directions $(+,-, 2,0)=(+,-, x, y)$, such that their worldvolume lies partly in only one of the $(4+$ 4)-dimensional transverse spaces. They are supported by an additional 5-form flux $F_{+-x y r}(r)$ where $r$ is the radius of the four-dimensional transverse space which does not include the $(x, y)$ directions, and are partially smeared since all metric components with the exception of $g_{++}$depend only on $r$. The D3 $(+,-, 2,0)$ branes preserve $1 / 4$ of the linearly realized supersymmetries. An ansatz which captures these features and generalizes (3.18) is

$$
\begin{aligned}
& \mathrm{d} s^{2}= \mathrm{e}^{2 A(r)}[- \\
&\left.4 \mathrm{~d} x^{+} \mathrm{d} x^{-}+\mathcal{H}(r, x, y, w, z) \mathrm{d} x^{+2}\right] \\
&+\mathrm{e}^{2 B(r)}\left[\mathrm{d} r^{2}+r^{2} \mathrm{~d} \Omega_{3}^{2}\right]+\mathrm{e}^{2 C(r)}\left(\mathrm{d} x^{2}+\mathrm{d} y^{2}\right)+\mathrm{e}^{2 D(r)}\left(\mathrm{d} w^{2}+\mathrm{d} z^{2}\right), \\
& F_{5}^{(0)}=2(1+*) \mathrm{d} x^{+} \wedge \mathrm{d} x^{-} \wedge \mathrm{d} x \wedge \mathrm{d} y \wedge \mathrm{de} \mathrm{e}^{2 E(r)}, \\
& F_{5}^{(1)}=\mu \mathrm{d} x^{+} \wedge\left(\mathrm{d} x \wedge \mathrm{d} y \wedge \mathrm{d} w \wedge \mathrm{d} z+\mathrm{e}^{4 B-2 C-2 D} r^{3} \mathrm{~d} r \wedge \mathrm{d} \Omega_{3}\right) .
\end{aligned}
$$

As in the D3 $(+,-, 1,1)$ case, the Einstein equations separate cleanly into a set of standard D3-brane equations and an additional Laplace equation for $\mathcal{H}$.

By taking appropriate linear combinations of Einstein equations such that the source terms cancel, we learn that

$$
A+B+C+D=\frac{1}{2} \mathcal{G}=\frac{1}{2} \log g(r), \quad A+D=\delta \log f(r), \quad A-C=\gamma \log f(r),
$$

where, as we have become accustomed to, the functions $g(r)$ and $f(r)$ have the same universal expression sensitive only to the dimensionality and particular splitting of the transverse space

$$
g(r)=1-\left(\frac{r_{0}}{r}\right)^{4}, \quad f(r)=\frac{1-\left(r_{0} / r\right)^{2}}{1+\left(r_{0} / r\right)^{2}} .
$$

The only nontrivial Bianchi identity yields

$$
\left(r^{3} \mathrm{e}^{-2 A+2 B-2 C+2 D}\left(\mathrm{e}^{2 E}\right)^{\prime}\right)^{\prime}=0 .
$$

Integrating this equation once, one can substitute it into the remaining Einstein equation,

$$
\left[\left(\mathrm{e}^{-4 \hat{A}}\right)^{\prime}\right]^{2}-\lambda^{2}\left(\mathrm{e}^{-4 \hat{A}}\right)^{2}\left(\frac{f^{\prime}}{f}\right)^{2}=4 \frac{L^{4}}{r^{6} g^{2}},
$$

where

$$
\hat{A}=A-\frac{\gamma}{2} \log f
$$


and

$$
\lambda^{2}=3-8 \delta^{2}+8 \delta \gamma-4 \gamma^{2} .
$$

The solution is again of the form

$$
\mathrm{e}^{-4 \hat{A}}=H \equiv \frac{L^{2}}{2 \lambda r_{0}^{2}} \sinh (c-\lambda \log f),
$$

with $c$ chosen so that $H \rightarrow 1$ as $r \rightarrow \infty$. As a result, the non-extremal D3 $(+,-, 2,0)$ metric has the form

$$
\begin{aligned}
\mathrm{d} s^{2}=H^{-\frac{1}{2}} f^{\gamma} & {\left[-4 \mathrm{~d} x^{+} \mathrm{d} x^{-}+\mathcal{H} \mathrm{d} x^{+2}+f^{-2 \gamma}\left(\mathrm{d} x^{2}+\mathrm{d} y^{2}\right)\right] } \\
+ & H^{\frac{1}{2}} f^{\gamma-2 \delta}\left[g\left(\mathrm{~d} r^{2}+r^{2} \mathrm{~d} \Omega_{3}^{2}\right)+f^{-2 \gamma+4 \delta}\left(\mathrm{d} w^{2}+\mathrm{d} z^{2}\right)\right],
\end{aligned}
$$

and reduces to the extremal case (3.18) with $H=1+L^{2} / r^{2}$, when $r_{0}=0$. The plane wave function $\mathcal{H}$ can be solved from the (++) Einstein equation

$$
\widehat{\square} \mathcal{H}+\mathrm{e}^{-2 C} \square_{x, y} \mathcal{H}+\mathrm{e}^{-2 D} \square_{w, z} \mathcal{H}=-\mu^{2} \mathrm{e}^{-2 A-4 C-4 D} .
$$

The resulting behaviour at $r_{0}$ is generically logarithmic, unless $|\lambda|+6 \delta-4 \gamma>3$, whereupon $\mathcal{H}$ would instead be dominated by a power law singularity, $\mathcal{H} \sim\left(r-r_{0}\right)^{-[\lambda \mid+6 \delta-4 \gamma-3]}$.

Extracting the behaviour of the warp factors near $r_{0}$, we find the horizon conditions

$$
\gamma-\delta>-\frac{3}{2}, \quad-|\lambda|+2 \delta \geqslant 3,
$$

(provided $\mathcal{H}$ is at most logarithmic at $r_{0}$ ). These conditions, in conjunction with (6.40), do not admit real solutions for $\gamma$ and $\delta$. Note, furthermore, that the case where $\mathcal{H}$ develops a power law behaviour imposes even stronger inequalities, and the solution is again singular.

6.2.3. The D1-D5 system. Finally, we note that the D1-D5 system examined in section 3.2.3 also admits a non-extremal generalization. The techniques for obtaining such a solution are similar to those for the cases considered above. As a result, we will be brief, and will only highlight the salient features of the solution.

The equations of motion governing the D1-D5 system were previously given in (3.22), and admitted the extremal solution (3.23). This solution may be generalized by introducing additional blackening functions in the metric

$\mathrm{d} s^{2}=H_{1}^{-\frac{3}{4}} H_{5}^{-\frac{1}{4}} f^{2 \alpha}\left[-4 \mathrm{~d} x^{+} \mathrm{d} x^{-}+\mathcal{H} \mathrm{d} x^{+2}\right]+H_{1}^{\frac{1}{4}} H_{5}^{-\frac{1}{4}} f^{2 \gamma} \mathrm{d} \vec{y}^{2}+H_{1}^{\frac{1}{4}} H_{5}^{\frac{3}{4}} f^{2 \beta} g \mathrm{~d} \vec{z}^{2}$,

where

$$
g(r)=1-\left(\frac{r_{0}}{r}\right)^{4}, \quad f(r)=\frac{1-\left(r_{0} / r\right)^{2}}{1+\left(r_{0} / r\right)^{2}},
$$

with $r=|\vec{z}|^{2}$. The constants $\alpha, \beta$ and $\gamma$ are then chosen to satisfy $2 \alpha+2 \beta+4 \gamma=0$, so that $\mathcal{G}$ defined after (3.7) takes on the canonical form $\mathcal{G}=\log g$.

As is well known, the D1 and D5 charges may become renormalized in the non-extremal case. Thus, we can no longer assume the RR 3-form to be given in terms of the same $H_{1}$ and $H_{5}$ functions that appear in the metric. We find the proper generalization of $F_{3}$ given in (3.23) to be

$$
F_{3}=-2 H_{1}^{-2} f^{2 \delta} g^{-1} \mathrm{~d} x^{+} \wedge \mathrm{d} x^{-} \wedge \mathrm{d}\left(\frac{Q_{1}}{r^{2}}\right)+\frac{1}{6} \epsilon_{i j k l} \partial_{l}\left(\frac{Q_{5}}{r^{2}}\right) \mathrm{d} z^{i} \wedge \mathrm{d} z^{j} \wedge \mathrm{d} z^{k},
$$

where $\delta$ is a constant to be determined. This ansatz along with the $F_{3}$ equation of motion then determines the dilaton to be

$$
\mathrm{e}^{\phi}=H_{1}^{\frac{1}{2}} H_{5}^{-\frac{1}{2}} f^{4 \alpha-2 \delta} .
$$


The remaining equations to be solved are the Einstein and dilaton equations of motion.

Solving the remaining equations yields the explicit form of $H_{1}$ and $H_{5}$,

$H_{1}=\frac{Q_{1}}{2 \lambda_{1} r_{0}^{2}} \sinh \left(c_{1}-\lambda_{1} \log f\right), \quad H_{5}=\frac{Q_{5}}{2 \lambda_{5} r_{0}^{2}} \sinh \left(c_{5}-\lambda_{5} \log f\right)$,

as well as the constraints $\alpha=\beta, \gamma=0$ and

$$
\lambda_{1}^{2}+\lambda_{5}^{2}+32 \alpha^{2}=3
$$

The constants $c_{1}$ and $c_{5}$ are chosen so that $H_{1}, H_{5} \rightarrow 1$ as $r \rightarrow \infty$. In this case, the "harmonic functions' have asymptotic behaviour

$$
H_{1}=1+\frac{\sqrt{Q_{1}^{2}+4 \lambda_{1}^{2} r_{0}^{4}}}{r^{2}}+\cdots, \quad H_{5}=1+\frac{\sqrt{Q_{5}^{2}+4 \lambda_{5}^{2} r_{0}^{4}}}{r^{2}}+\cdots,
$$

indicating explicitly the effect of charge renormalization. On the other hand, near $r_{0}$, the functions behave as

$$
H_{1} \sim\left(r-r_{0}\right)^{-\left|\lambda_{1}\right|}, \quad H_{5} \sim\left(r-r_{0}\right)^{-\left|\lambda_{5}\right|} .
$$

Collecting the above results, we find the non-extremal generalization of the D1-D5 solution to be

$$
\begin{aligned}
& \mathrm{d} s^{2}=H_{1}^{-\frac{3}{4}} H_{5}^{-\frac{1}{4}} f^{2 \alpha}\left[-4 \mathrm{~d} x^{+} \mathrm{d} x^{-}+\mathcal{H} \mathrm{d} x^{+2}\right]+H_{1}^{\frac{1}{4}} H_{5}^{-\frac{1}{4}} f^{-2 \alpha} \mathrm{d} \vec{y}^{2}+H_{1}^{\frac{1}{4}} H_{5}^{\frac{3}{4}} f^{2 \alpha} g \mathrm{~d} \vec{z}^{2}, \\
& F_{5}=\mu \mathrm{d} x^{+} \wedge\left(\mathrm{d} y^{1} \wedge \mathrm{d} y^{2} \wedge \mathrm{d} z^{1} \wedge \mathrm{d} z^{2}+\mathrm{d} y^{3} \wedge \mathrm{d} y^{4} \wedge \mathrm{d} z^{3} \wedge \mathrm{d} z^{4}\right), \\
& F_{3}=-2 H_{1}^{-2} g^{-1} \mathrm{~d} x^{+} \wedge \mathrm{d} x^{-} \wedge \mathrm{d}\left(\frac{Q_{1}}{r^{2}}\right)+\frac{1}{6} \epsilon_{i j k l} \partial_{l}\left(\frac{Q_{5}}{r^{2}}\right) \mathrm{d} z^{i} \wedge \mathrm{d} z^{j} \wedge \mathrm{d} z^{k}, \\
& \mathrm{e}^{2 \phi}=H_{1} H_{5}^{-1} f^{8 \alpha},
\end{aligned}
$$

where $H_{1}$ and $H_{5}$ are given by (6.49), and $\mathcal{H}$ satisfies

$$
r^{-3} \partial_{r}\left(r^{3} g \partial_{r} \mathcal{H}\right)+H_{5} f^{4 \alpha} g^{2} \widetilde{\square} \mathcal{H}=-\mu^{2},
$$

which limits to (3.25) when $r_{0} \rightarrow 0$. Again, proper asymptotics may be obtained by setting $\mathcal{H}=-\frac{1}{16} \mu^{2}\left(r^{2}+\vec{y}^{2}\right)+\overline{\mathcal{H}}(r)$ where

$$
r^{-3} \partial_{r}\left(r^{3} g \partial_{r} \overline{\mathcal{H}}\right)=\frac{1}{2} \mu^{2}\left(H_{5} f^{4 \alpha} g^{2}-1\right) .
$$

Turning to the issue of horizons, we find that

$$
\mathrm{e}^{2 A} \sim\left(r-r_{0}\right)^{\frac{3}{4}\left|\lambda_{1}\right|+\frac{1}{4}\left|\lambda_{5}\right|+2 \alpha}, \quad \mathrm{e}^{2 B} \sim\left(r-r_{0}\right)^{-\frac{1}{4}\left|\lambda_{1}\right|-\frac{3}{4}\left|\lambda_{5}\right|+2 \alpha+1},
$$

in the language of section 2 . Thus the conditions for the existence of a horizon are

$$
\left|\lambda_{1}\right|-\left|\lambda_{5}\right|+8 \alpha>-6, \quad\left|\lambda_{1}\right|+\left|\lambda_{5}\right| \geqslant 3 .
$$

The second inequality is incompatible with the ellipsoidal constraint (6.50). Hence the solution is a naked singularity. Note that in (6.57) we have implicitly assumed that $\mathcal{H}$ develops at most a logarithmic singularity. Since no horizon is possible in this case, neither can it be possible for the case where $\mathcal{H}$ has a power law behaviour. Thus, once more, in attempting to nonextremalize well-behaved solutions, we instead end up with a naked singularity at $r_{0}$.

While these string, D3 and D1-D5 examples have not been exhaustive, they nevertheless suggest that the essence of the no-go theorem proved in the previous section continues to apply, even in the presence of additional matter sources. The common feature in all these cases is of course the null Killing vector, whose preservation requires a simultaneous blackening of two longitudinal directions. This results in an 'overblackened' solution, which is necessarily a naked singularity. Thus it is likely that one must give up the null Killing vector in order to avoid the appearance of such singularities. This will be examined in the following section. 


\section{Relaxing the null Killing vector condition}

Until now, we have focused on metrics admitting a null Killing vector as the simplest generalization allowing a horizon and capturing some of the features of the BFHP solution. However, as we have seen, both the no-go theorem of section 5 (in the absence of sources) and the examples of section 6 (with additional sources turned on) suggest that the null Killing vector condition may still be too restrictive, at least for obtaining a non-extremal solution with regular horizon. This difficulty arises in a way since the null Killing vector, being $\partial / \partial x^{-}$, is not obviously related to the null direction in $(t, r)$ that would exist at the horizon of, e.g., a Schwarzschild black hole. As a result, this incompatibility of symmetries apparently prevents the existence of a regular horizon. To proceed, it is clear that we must relax the null Killing vector condition. However, to maintain some relation to the BFHP solution, we must ensure that proper boundary conditions are satisfied so that the geometry may at least asymptotically be regarded as the BFHP solution.

Let us further consider the general structure of the solution we are seeking. The general solution would naturally contain two scales: $\mu$, giving the asymptotic strength of the RR 5 -form, and $r_{0}$, essentially the Schwarzschild radius or non-extremality scale. Finding an explicit solution for arbitrary values of $r_{0}$ and $\mu$ has proved to be a difficult task. However, we may consider various limiting cases where we have a rough intuition of what the solution should look like. For example, in the limiting case $r_{0} \mu \ll 1$, the two main scales are widely separated. This limit is essentially that of a small black hole in the plane wave background ${ }^{6}$.

With this separation of scales, if we focus on the region $r \gg \mu^{-1}$, the details of the black hole ought to be irrelevant, and the metric should end up being asymptotic to the plane wave (3.10). On the other hand, in the region where $r \ll \mu^{-1}$, the black hole geometry dominates, and the 5-form flux supporting the background may be considered as a perturbation to the Schwarzschild black hole. This is precisely the limit we consider here. We thus will examine the consistency of this solution by exploring the possibility of perturbatively turning on a 5 -form in a Schwarzschild-like metric. Namely, we begin by considering a black string in IIB theory which is the natural starting point (due to the symmetries) for the plane wave.

We find it convenient to work in isotropic coordinates. The black string in ten dimensions may be written as

$$
\mathrm{d} s^{2}=-f^{2} \mathrm{~d} t^{2}+\mathrm{d} y^{2}+h^{\frac{2}{3}} \mathrm{~d} \vec{z}^{2}
$$

where

$$
f=\frac{1-\left(r_{0} / r\right)^{6}}{1+\left(r_{0} / r\right)^{6}}, \quad h=1+\left(\frac{r_{0}}{r}\right)^{6} .
$$

Here, $r=|\vec{z}|$ is an eight-dimensional transverse coordinate. By defining $y=x^{+}-2 x^{-}$and $t=2 x^{-}$, the black string metric may be written in the equivalent form

$$
\mathrm{d} s^{2}=-4 \mathrm{~d} x^{+} \mathrm{d} x^{-}+\mathrm{d} x^{+2}+4\left(1-f^{2}\right) \mathrm{d} x^{-2}+h^{\frac{2}{3}} \mathrm{~d} \vec{z}^{2} .
$$

In the limit $r \rightarrow \infty$ we see that $f \rightarrow 1$ and therefore $g_{--} \rightarrow 0$. Hence $\partial / \partial x^{-}$, which is a Killing vector, becomes asymptotically null. This allows us to make connection with the plane wave at infinity.

We now seek to turn on an RR 5-form in the background (7.3). We first note that no solution is possible that maintains the accidental SO(8) symmetry. This is easily seen by

6 The situation is similar to the small black hole in AdS. Although the existence of this small black hole is conceptually clear, finding an explicit solution has not been possible so far [34]. The main obstruction is the same symmetry considerations we face here. 
writing the most general 5 -form ansatz compatible with both this symmetry and self-duality as

$$
\begin{aligned}
& F_{5}=\left(f_{1} \mathrm{~d} x^{+}-2\left(f_{1}+f_{2} f\right) \mathrm{d} x^{-}\right) \wedge \mathrm{d} z^{1} \wedge \mathrm{d} z^{2} \wedge \mathrm{d} z^{3} \wedge \mathrm{d} z^{4} \\
&+\left(f_{2} \mathrm{~d} x^{+}-2\left(f_{2}+f_{1} f\right) \mathrm{d} x^{-}\right) \wedge \mathrm{d} z^{5} \wedge \mathrm{d} z^{6} \wedge \mathrm{d} z^{7} \wedge \mathrm{d} z^{8},
\end{aligned}
$$

where $f_{1}(r)$ and $f_{2}(r)$ are arbitrary functions of $r$. The Bianchi identity $\mathrm{d} F_{5}=0$ then demands that $f_{1}, f_{2}, f_{1} f$ and $f_{2} f$ are all constant. For $r_{0}=0(f=1)$, this allows the solution (3.10). However, for $r_{0} \neq 0$, this is only compatible with the complete vanishing of $F_{5}$. We have thus demonstrated that the non-extremal solution cannot maintain the accidental $S O(8)$ invariance of the metric. This should not come as a surprise, because once we abandon the null Killing vector $\partial / \partial x^{-}$, we essentially return to the Freund-Rubin case, where the 5 -form is responsible for the negative and positive curvatures of $\mathrm{AdS}_{5} \times S^{5}$.

Of course, the $S O(8)$ symmetry of (3.10) was never a true symmetry in the first place. So there is no difficulty in giving it up. However, we expect to still retain the $S O(4) \times S O(4)$ symmetry. To do so, we write the transverse metric as

$$
\mathrm{d} \vec{z}^{2}=\mathrm{d} r^{2}+r^{2}\left[\mathrm{~d} \theta^{2}+\sin ^{2} \theta \mathrm{d} \Omega_{3}^{2}+\cos ^{2} \theta \mathrm{d} \widetilde{\Omega}_{3}^{2}\right] .
$$

The most general 5 -form allowed by symmetry and self-duality is now considerably more complicated. Following [34], we determine

$$
\begin{aligned}
F_{5}=g_{4} r^{3} \sin ^{4} \theta \mathrm{d} x^{+} \wedge \mathrm{d} r \wedge \mathrm{d} \Omega_{3}+g_{3} r^{4} \sin ^{3} \theta \cos \theta \mathrm{d} x^{+} \wedge \mathrm{d} \theta \wedge \mathrm{d} \Omega_{3} \\
+g_{2} r^{3} \cos ^{4} \theta \mathrm{d} x^{+} \wedge \mathrm{d} r \wedge \mathrm{d} \widetilde{\Omega}_{3}-g_{1} r^{4} \cos ^{3} \theta \sin \theta \mathrm{d} x^{+} \wedge \mathrm{d} \theta \wedge \mathrm{d} \widetilde{\Omega}_{3} \\
+2\left(g_{1} f-g_{4}\right) r^{3} \sin ^{4} \theta \mathrm{d} x^{-} \wedge \mathrm{d} r \wedge \mathrm{d} \Omega_{3} \\
+2\left(g_{2} f-g_{3}\right) r^{4} \sin ^{3} \theta \cos \theta \mathrm{d} x^{-} \wedge \mathrm{d} \theta \wedge \mathrm{d} \Omega_{3} \\
+2\left(g_{3} f-g_{2}\right) r^{3} \cos ^{4} \theta \mathrm{d} x^{-} \wedge \mathrm{d} r \wedge \mathrm{d} \widetilde{\Omega}_{3} \\
\\
-2\left(g_{4} f-g_{1}\right) r^{4} \cos ^{3} \theta \sin \theta \mathrm{d} x^{-} \wedge \mathrm{d} \theta \wedge \mathrm{d} \widetilde{\Omega}_{3},
\end{aligned}
$$

where $g_{i}(r, \theta), i=1,2,3,4$ are, in principle, functions of both $r$ and $\theta$. After suitable manipulation, the Bianchi identities read

$$
\begin{aligned}
& \left(-\cot \theta \partial_{\theta}+4\right) g_{2}=\left(r \partial_{r}+4\right) g_{1}, \\
& \left(\tan \theta \partial_{\theta}+4\right) g_{1}=\left(r \partial_{r}+r \partial_{r} \log f+4\right) g_{2}, \\
& \left(\tan \theta \partial_{\theta}+4\right) g_{4}=\left(r \partial_{r}+4\right) g_{3}, \\
& \left(-\cot \theta \partial_{\theta}+4\right) g_{3}=\left(r \partial_{r}+r \partial_{r} \log f+4\right) g_{4} .
\end{aligned}
$$

Note that this splits into two pairs of equations, one for $g_{1}, g_{2}$ and another identical set for $g_{3}, g_{4}$.

In the extremal case $(f=1)$, the Bianchi identities (which are the 5 -form equations of motion) have a trivial solution $g_{i}=\mu, i=1,2,3,4$. This yields precisely the 5 -form of (3.10). More generally, we wish to retain this asymptotic behaviour, $g_{i} \rightarrow \mu$ as $f \rightarrow 1$, but otherwise solve (7.7) for $f$ of the form (7.2). Concentrating on $g_{1}, g_{2}$, the first two lines of (7.7) may be differentiated further to obtain the second-order equations

$$
\begin{aligned}
& \left(\frac{1}{r^{9} f} \partial_{r} r^{9} f \partial_{r}+\frac{1}{r^{2} \sin ^{5} \theta \cos ^{3} \theta} \partial_{\theta} \sin ^{5} \theta \cos ^{3} \theta \partial_{\theta}+\frac{4}{r} \partial_{r} \log f\right) g_{1}(r, \theta)=0, \\
& \left(\frac{1}{r^{9} f} \partial_{r} r^{9} f \partial_{r}+\frac{1}{r^{2} \sin ^{3} \theta \cos ^{5} \theta} \partial_{\theta} \sin ^{3} \theta \cos ^{5} \theta \partial_{\theta}+\frac{5}{r} \partial_{r} \log f+\partial_{r}^{2} \log f\right) g_{2}(r, \theta)=0 .
\end{aligned}
$$




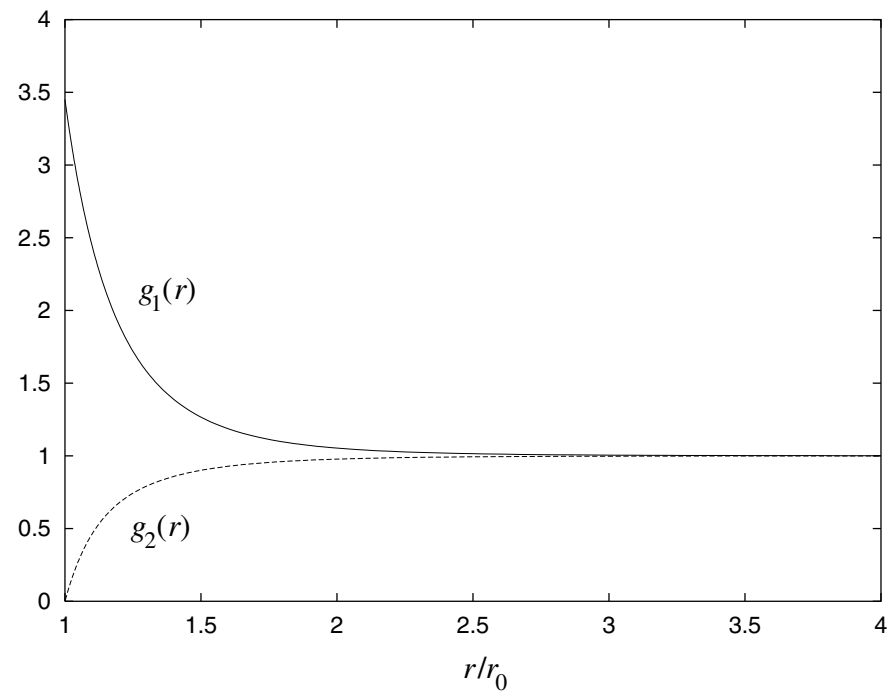

Figure 1. The functions $g_{1}$ and $g_{2}$ normalized such that $g_{i} \rightarrow 1$ as $r \rightarrow \infty$. Note that $g_{2}$ vanishes at the horizon.

These are simply the second-order equations of motion for the 5-form. In general, solutions do exist compatible with the asymptotic condition $g_{i} \rightarrow \mu$ as $r \rightarrow \infty$. However, for simplicity, we now assume that $g_{i}(r)$ are independent of $\theta$. In this case, we only need to solve

$$
\left(\frac{1}{r^{9} f} \partial_{r} r^{9} f \partial_{r}+\frac{4}{r} \partial_{r} \log f\right) g_{1}(r)=0 .
$$

Then $g_{2}$ may be obtained from the first equation of (7.7), namely $g_{2}(r)=\left(1+\frac{1}{4} r \partial_{r}\right) g_{1}(r)$.

Since (7.9) is a linear second-order equation, it admits two solutions. It is easy to see that as $r \rightarrow \infty$ the solutions have behaviour $g_{1} \sim 1$ and $g_{1} \sim r^{-8}$. The former yields the proper asymptotics, while the latter falls off faster than the blackening function and is subdominant. On the other hand, the horizon behaviour of $g_{1}$ is governed by the limit $f \rightarrow 0$ and may be obtained by expanding (7.9) for $r \rightarrow r_{0}$. Near the horizon, we find either $g_{1} \sim 1$ or $g_{1} \sim \log \left(r-r_{0}\right)$, so that one solution remains finite, while the second blows up. Keeping only the solution that remains finite at the horizon, this gives a unique $\theta$-independent solution for the $g_{i}$, up to overall normalization. The numerical solution to (7.9) which satisfies such boundary conditions is shown in figure 1 . As a result, this demonstrates that the 5-form with the desired asymptotics for connecting to the plane wave of (3.10) may be consistently turned on in the black string background.

Although we do not compute the backreaction from turning on the 5-form, it is nevertheless instructive to examine the stress-energy source. For the 5-form of (7.6), we find

$$
\begin{aligned}
& F_{++}^{2}=48 h^{-\frac{8}{3}} G_{+}^{2}, \\
& F_{+-}^{2}=96 h^{-\frac{8}{3}}\left(g_{1} g_{4} f-G_{+}^{2}\right), \\
& F_{--}^{2}=192 h^{-\frac{8}{3}}\left(G_{+}^{2}\left(1+f^{2}\right)-2 g_{1} g_{2} f\right),
\end{aligned}
$$


for the longitudinal directions, and

$$
\begin{aligned}
& F_{r r}^{2}=48 h^{-2} G_{-}^{2}, \\
& F_{\theta \theta}^{2}=-48 h^{-2} r^{2} G_{-}^{2}, \\
& F_{\alpha \beta}^{2}=48 h^{-2} r^{2} \sin ^{2} \theta g_{\alpha \beta} G_{-}^{2}\left(\sin ^{2} \theta-\cos ^{2} \theta\right), \\
& F_{\tilde{\alpha} \tilde{\beta}}^{2}=-48 h^{-2} r^{2} \cos ^{2} \theta g_{\tilde{\alpha} \tilde{\beta}} G_{-}^{2}\left(\sin ^{2} \theta-\cos ^{2} \theta\right),
\end{aligned}
$$

for the transverse ones. Here, for convenience, we have defined $G_{ \pm}^{2} \equiv \frac{1}{2}\left(g_{4}^{2} \pm g_{1}^{2}\right)$. The asymptotic behaviour is guaranteed as $G_{-}^{2} \rightarrow 0$ as $r \rightarrow \infty$, while all quantities remain finite at the horizon. This suggests that the backreaction is under control. Furthermore, we see explicitly that the stress-energy source in the transverse directions will break the $S O(8)$ symmetry of the metric (7.3), once backreaction is taken into account. While we have not obtained a complete nonlinear solution, this analysis in the regime $r_{0} \mu \ll 1$ provides strong evidence that an appropriate non-extremal gravitational background does exist.

It is worth mentioning that there are other approaches for understanding the phase diagram of asymptotically BFHP solutions. A natural attempt, complementary to that presented in this section, would be to consider the motion of a particle in the plane wave background and the subsequent effects on the geometry of increasing the mass of the particle ${ }^{7}$. This approach has been recently discussed in [35].

The complete understanding of phase transitions (like the Hawking-Page phase transition) and its gauge theory interpretation along the lines of [3] requires knowledge of the solution for arbitrary values of $r_{0}$ and $\mu$. It seems likely at this point that such a phase diagram might be more intricate than the corresponding one for asymptotically AdS spaces, in which case finding its complete structure would require a mixture of approaches. We believe, however, that our arguments for the existence of a solution with a well-defined Schwarzschild-type horizon add a crucial element to the understanding of the ultimate phase diagram.

\section{Conclusions}

In this paper, we have studied the existence of horizons in asymptotically plane wave spaces. We slightly generalized a generating solution mechanism due to Garfinkle and Vachaspati [15] (see also [16]) to include null matter. We applied this generating solution mechanism to flat space, 3-branes and intersecting branes. The case of 3-branes allows two possibilities, $\mathrm{D} 3(+,-, 1,1)$ and $\mathrm{D} 3(+,-, 2,0)$. Only the latter admits a deformation with a regular horizon. Unfortunately, this horizon has zero temperature. The $\mathcal{H}$ deformation of the D1/D5 system maintains a regular extremal horizon. We thus constructed two explicit examples where a extremal solution was deformed to asymptote to the maximally supersymmetric plane wave while preserving an extremal horizon.

Various attempts at deforming non-extremal solutions to asymptote to a plane wave yielded naked singularities. In particular, we considered $S O(8)$ and $S O(4) \times S O(4)$ symmetries of the transverse space. We proved a no-go theorem stating that in the presence of null matter and a null Killing vector there cannot be a smooth non-zero temperature horizon. The explicit introduction of different sets of sources did not result in a smooth non-zero temperature horizon.

We provided a perturbative argument in favour of the existence of smooth non-zero temperature horizons by considering a deformation of the black string in ten dimensions. We showed that the backreaction is under control.

\footnotetext{
7 We thank A Hashimoto and E Gimon for discussions of this possibility.
} 
In this paper, we have provided ample evidence for the fact that the existence of a null Killing vector (not necessarily covariantly constant) is an obstruction to the existence of a smooth non-zero temperature horizon. We have also shown perturbatively that once the null Killing vector is removed the possibility of a smooth non-zero temperature horizon arises.

\section{Acknowledgments}

We would like to thank A Hashimoto, I Klebanov, F Larsen, J Maldacena, D Marolf and R Roiban. We are especially grateful to E Gimon for a very fruitful conversation. JTL was supported in part by DOE grant DE-FG02-95ER40899. LAPZ was supported by a grant in aid from the Funds for Natural Sciences at IAS. The work of DV is supported by DOE grant DE-FG02-91ER40671.

\section{Appendix A. Other asymptotic times}

For spacetimes asymptotic to the plane wave, we have been able to identify the asymptotic time with $x^{+}$. This is, however, not the case for various examples of known solutions which have a null Killing vector and a regular horizon. To clarify this point and to warn the reader that the horizon criteria must be applied with care, we will present two examples where (2.13) is not directly applicable.

The first example is the five-dimensional (5D) three charge black hole solution. When lifted to ten dimensions it is mapped into a D1-D5 system (in the string frame)

$\mathrm{d} s^{2}=\left(H_{1} H_{5}\right)^{-1 / 2}\left(-\mathrm{d} x^{+} \mathrm{d} x^{-}+K \mathrm{~d} x^{+2}\right)+\left(H_{1} H_{5}\right)^{1 / 2} \mathrm{~d} \vec{x}_{4}^{2}+H_{1}^{1 / 2} H_{5}^{-1 / 2} \mathrm{~d} \vec{y}_{4}^{2}$,

where the three charges of the 5D black hole correspond to the number of D1-branes, $Q_{1}$, the number of D5-branes, $Q_{5}$, and the momentum along the compactified ninth direction, $N$,

$$
H_{1}(r)=1+\frac{c_{1} Q_{1}}{r^{2}}, \quad H_{5}(r)=1+\frac{c_{5} Q_{5}}{r^{2}}, \quad K(r)=\frac{c_{w} N}{r^{2}}
$$

and where $r^{2}=|\vec{x}|^{2}$. Given that this solution asymptotes to Minkowski flat space, we may choose

$$
t=\frac{x^{+}+x^{-}}{2}, \quad x_{9}=\frac{x^{-}-x^{+}}{2},
$$

(or a similar but boosted frame). The clock of the observer at infinity measures time in the coordinate $t$ rather than $x^{+}$, as would be natural for the case of solutions asymptoting to BFHP. Using the two integrals of motion

$E=\left(H_{1} H_{5}\right)^{-1 / 2}\left((1-K) \dot{t}+K \dot{x}_{9}\right), \quad E_{9}=\left(H_{1} H_{5}\right)^{-1 / 2}\left((1+K) \dot{x}_{9}-K \dot{t}\right)$,

the radial timelike geodesic, in the vicinity of $r=0$, is given by

$$
-1=\frac{1}{r^{2}}\left[-(1+K) E^{2}+(1-K) E_{9}^{2}+2 K E E_{9}\right]+\frac{\dot{r}^{2}}{r^{2}} .
$$

Hence the proper time spent near the horizon is finite,

$$
\int \mathrm{d} \tau \sim \int r \mathrm{~d} r
$$

while, from the point of view of the observer at infinity, the time elapsed is infinite,

$$
\int \mathrm{d} t \sim \int \frac{\mathrm{d} r}{r}
$$


These results are a particular case of (2.12), where we must consider that near $r=0$ it is $\Delta x^{-}$ which provides the leading behaviour of $\Delta t$.

The area of the horizon is finite, and the entropy is solely determined by the product of the three charges, $S=A_{H} / 4 G_{N}=2 \pi \sqrt{N Q_{1} Q_{5}}$. Note that for this solution the norm of the Killing vector $\xi=\partial / \partial t$ is vanishing on the ergosphere, $1-K\left(r_{E}\right)=0$, and is positive for $0 \leqslant r \leqslant r_{E}$. A similar situation was encountered for the four-dimensional charged Kerr black hole. This means only that to pass through the stationary surface at $r_{E}$ following a timelike radial geodesic, one needs motion in the $x_{9}$ direction as well; i.e., setting $\dot{x}_{9}=0$ would be inconsistent.

The second example that we want to discuss is the 5D black string obtained in [22] (see also $[16,23])$. This five-dimensional solution, whose metric in the string frame is given by

$$
\mathrm{d} s^{2}=\frac{f}{h} \mathrm{~d} x^{+2}+\frac{2}{h} \mathrm{~d} x^{+} \mathrm{d} x^{-}+k l\left(\mathrm{~d} r^{2}+r^{2} \mathrm{~d} \Omega_{3}^{2}\right)
$$

is characterized by four charges

$$
\begin{array}{ll}
f(r)=1+\frac{Q_{1}}{r}, & h(r)=1+\frac{Q_{2}}{r}, \\
k(r)=1+\frac{P_{1}}{r}, & l(r)=1+\frac{P_{2}}{r} .
\end{array}
$$

The asymptotic metric is Minkowski, and the asymptotic time is naturally identified as

$$
t=x^{-}, \quad x_{5}=x^{+}+x^{-}
$$

(again up to a boost). The event horizon is located at $r=0$, as we can convince ourselves by estimating the proper time elapsed as the free falling particle approaches the horizon

$$
-1=-\frac{E_{-}^{2}}{r^{2}}+2 \frac{E_{-} E_{+}}{r}+\frac{\dot{r}^{2}}{r^{2}}
$$

where $E_{-}, E_{+}$are integrals of motion associated with the $\partial / \partial x^{-}, \partial / \partial x^{+}$isometries. Thus the proper time is finite, $\int \mathrm{d} \tau \sim \int \mathrm{d} r$, while the observer at infinity measures an infinite time, $\int \mathrm{d} t \sim \int r^{-2} \mathrm{~d} r$. As before, there is a stationary surface where $g_{t t}$ vanishes, and to reach the horizon at $r=0$ the free falling particles must move in the $x_{5}$ direction as well.

\section{References}

[1] Maldacena J 1998 The large $N$ limit of superconformal field theories and supergravity Adv. Theor. Math. Phys. 2231 (Preprint hep-th/9711200)

Gubser S S, Klebanov I R and Polyakov A M 1998 Gauge theory correlators from non-critical string theory Phys. Lett. B 428105 (Preprint hep-th/9802109)

Witten E 1998 Anti-de Sitter space and holography Adv. Theor. Math. Phys. 253 (Preprint hep-th/9802150)

[2] Hawking S W and Page D N 1983 Thermodynamics of black holes in anti-de Sitter space Commun. Math. Phys. 87577

[3] Witten E 1998 Anti-de Sitter space, thermal phase transition, and confinement in gauge theories Adv. Theor. Math. Phys. 2505 (Preprint hep-th/9803131)

[4] Chamblin A, Emparan R, Johnson C V and Myers R C 1999 Charged AdS black holes and catastrophic holography Phys. Rev. D 60064018 (Preprint hep-th/9902170)

Chamblin A, Emparan R, Johnson C V and Myers R C 1999 Large $N$ phases, gravitational instantons and the nuts and bolts of AdS holography Phys. Rev. D 59064010 (Preprint hep-th/9808177)

[5] Berenstein D, Maldacena J M and Nastase H 2002 Strings in flat space and pp waves from $\mathcal{N}=4$ super Yang-Mills J. High Energy Phys. JHEP04(2002)013 (Preprint hep-th/0202021)

[6] Blau M, Figueroa-O'Farrill J, Hull C and Papadopoulos G 2002 A new maximally supersymmetric background of IIB superstring theory J. High Energy Phys. JHEP01(2002)047 (Preprint hep-th/0110242) 
[7] Kowalski-Glikman J 1984 Vacuum states in supersymmetric Kaluza-Klein theory Phys. Lett. B 134194

[8] Metsaev R R 2002 Type IIB Green-Schwarz superstring in plane wave Ramond-Ramond background Nucl. Phys. B 62570 (Preprint hep-th/0112044)

[9] Metsaev R R and Tseytlin A A 2002 Exactly solvable model of superstring in plane wave Ramond-Ramond background Phys. Rev. D 65126004 (Preprint hep-th/0202109)

Russo J G and Tseytlin A A 2002 On solvable models of type 2B superstring in NS-NS and R-R wave backgrounds J. High Energy Phys. JHEP04(2002)021 (Preprint hep-th/0202179)

[10] Pando Zayas L A and Vaman D 2003 Strings in RR plane wave background at finite temperature Phys. Rev. D 67106006 (Preprint hep-th/0208066)

Greene B R, Schalm K and Shiu G 2002 On the Hagedorn behaviour of pp-wave strings and $N=4$ SYM theory at finite $R$-charge density Preprint hep-th/0208163

Sugawara Y 2002 Thermal amplitudes in DLCQ superstrings on pp-waves Preprint hep-th/0209145

Brower R C, Lowe D A and Tan C I 2002 Hagedorn transition for strings on pp-waves and tori with chemical potentials Preprint hep-th/0211201

[11] Pando Zayas L A and Sonnenschein J 2002 On Penrose limits and gauge theories J. High Energy Phys. JHEP05(2002)010 (Preprint hep-th/0202186)

[12] Marolf D and Pando Zayas L A 2002 On the singularity structure and stability of plane waves Preprint hep-th/0210309

[13] Hubeny V E and Rangamani M 2002 No horizons in pp-waves J. High Energy Phys. JHEP11(2002)021 (Preprint hep-th/0210234)

[14] Hubeny V E and Rangamani M 2002 Generating asymptotically plane wave spacetimes Preprint hep-th/0211206

[15] Garfinkle D 1990 Traveling waves in strongly gravitating cosmic strings Phys. Rev. D 411112 Garfinkle D and Vachaspati T 1990 Cosmic string traveling waves Phys. Rev. D 421960 Garfinkle D 1992 Black string traveling waves Phys. Rev. D 464286 (Preprint gr-qc/9209002)

[16] Kaloper N, Myers R C and Roussel H 1997 Wavy strings: black or bright? Phys. Rev. D 557625 (Preprint hep-th/9612248)

[17] Wald R M 1984 General Relativity (Chicago, IL: University of Chicago Press)

[18] Berenstein D and Nastase H 2002 On lightcone string field theory from super Yang-Mills and holography Preprint hep-th/0205048

[19] Marolf D and Ross S F 2002 Plane waves: to infinity and beyond! Class. Quantum Grav. 196289 (Preprint hep-th/0208197)

[20] Hubeny V E and Rangamani M 2002 Causal structures of pp-waves Preprint hep-th/0211195

[21] Hayward S A 1994 General laws of black hole dynamics Phys. Rev. D 496467 (Preprint gr-qc/9309004)

[22] Cvetic M and Youm D 1996 Dyonic BPS saturated black holes of heterotic string on a six torus Phys. Rev. D 53 584 (Preprint hep-th/9507090)

[23] Cvetic M and Tseytlin A A 1996 General class of BPS saturated dyonic black holes as exact superstring solutions Phys. Lett. B 36695 (Preprint hep-th/9510097)

Cvetic M and Tseytlin A A 1996 Solitonic strings and BPS saturated dyonic black holes Phys. Rev. D 535619 (Preprint hep-th/9512031)

Cvetic M and Tseytlin A A 1997 Solitonic strings and BPS saturated dyonic black holes Phys. Rev. D 553907 (erratum)

[24] Cvetic M, Lu H and Pope C N 2002 Penrose limits, pp-waves and deformed M2-branes Preprint hep-th/0203082

[25] Bain P, Meessen P and Zamaklar M 2002 Supergravity solutions for D-branes in Hpp-wave backgrounds Preprint hep-th/0205106

[26] Alishahiha M and Kumar A 2002 D-brane solutions from new isometries of pp-waves Phys. Lett. B 542130 (Preprint hep-th/0205134)

[27] Skenderis K and Taylor M 2002 Branes in AdS and pp-wave spacetimes J. High Energy Phys. JHEP06(2002)025 (Preprint hep-th/0204054)

[28] Gaberdiel M R and Green M B 2002 The D-instanton and other supersymmetric D-branes in IIB plane-wave string theory Preprint hep-th/0211122

[29] Bain P, Peeters K and Zamaklar M 2002 D-branes in a plane wave from covariant open strings Preprint hep-th/0208038

[30] Skenderis K and Taylor M 2002 Open strings in the plane wave background: I. Quantization and symmetries Preprint hep-th/0211011

[31] Bergman O, Gaberdiel M R and Green M B 2002 D-brane interactions in type IIB plane-wave background Preprint hep-th/0205183

[32] Duff M J, Lu H and Pope C N 1996 The black branes of M-theory Phys. Lett. B 38273 (Preprint hep-th/9604052)

[33] Buchel A 2001 Finite temperature resolution of the Klebanov-Tseytlin singularity Nucl. Phys. B 600219 (Preprint hep-th/0011146) 
Buchel A, Herzog C P, Klebanov I R, Pando Zayas L A and Tseytlin A A 2001 Non-extremal gravity duals for fractional D3-branes on the conifold J. High Energy Phys. JHEP04(2002)033 Preprint hep-th/0102105

Gubser S S, Herzog C P, Klebanov I R and Tseytlin A A 2001 Restoration of chiral symmetry: a supergravity perspective J. High Energy Phys. JHEP05(2001)028 Preprint hep-th/0102172

[34] Horowitz G T and Hubeny V E 2000 Note on small black holes in $\operatorname{AdS}_{p} \times S^{q}$ J. High Energy Phys. JHEP06(2002)031 Preprint hep-th/0005288

[35] Li M 2002 Correspondence principle in a pp-wave background Nucl. Phys. B 638155 Preprint hep-th/0205043 Li M 2002 PP-wave black holes and the matrix model Preprint hep-th/0212345 